\title{
Controllability of Complex Networks at Minimum Cost
}

Gustav Lindmark 
Linköping studies in science and technology. Dissertations.

No. 2074

\section{Controllability of Complex Networks at Minimum Cost}

\section{Gustav Lindmark}

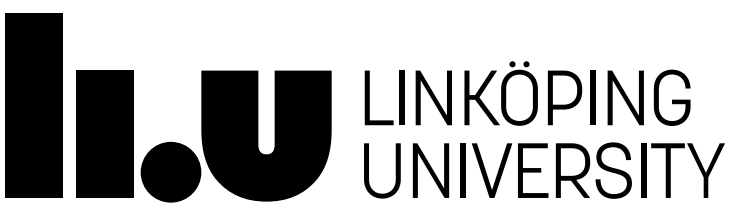


Linköping studies in science and technology. Dissertations.

No. 2074

Controllability of Complex Networks at Minimum Cost

Gustav Lindmark

gustav. Iindmarkeliu.se

ww. control.isy.liu.se

Division of Automatic Control

Department of Electrical Engineering

Linköping University

SE-581 83 Linköping

Sweden

ISBN 978-91-7929-847-0

ISSN 0345-7524

Copyright (C) 2020 Gustav Lindmark

Printed by LiU-Tryck, Linköping, Sweden 2020 
To my family 



\section{Abstract}

The control-theoretic notion of controllability captures the ability to guide a system toward a desired state with a suitable choice of inputs. Controllability of complex networks such as traffic networks, gene regulatory networks, power grids etc. can for instance enable efficient operation or entirely new applicative possibilities. However, when control theory is applied to complex networks like these, several challenges arise. This thesis considers some of them, in particular we investigate how a given network can be rendered controllable at a minimum cost by placement of control inputs or by growing the network with additional edges between its nodes. As cost function we take either the number of control inputs that are needed or the energy that they must exert.

A control input is called unilateral if it can assume either positive or negative values, but not both. Motivated by the many applications where unilateral controls are common, we reformulate classical controllability results for this particular case into a more computationally-efficient form that enables a large scale analysis. Assuming that each control input targets only one node (called a driver node), we show that the unilateral controllability problem is to a high degree structural: from topological properties of the network we derive theoretical lower bounds for the minimal number of unilateral control inputs, bounds similar to those that have already been established for the minimal number of unconstrained control inputs (e.g. can assume both positive and negative values). With a constructive algorithm for unilateral control input placement we also show that the theoretical bounds can often be achieved.

A network may be controllable in theory but not in practice if for instance unreasonable amounts of control energy are required to steer it in some direction. For the case with unconstrained control inputs, we show that the control energy depends on the time constants of the modes of the network, the longer they are, the less energy is required for control. We also present different strategies for the problem of placing driver nodes such that the control energy requirements are reduced (assuming that theoretical controllability is not an issue). For the most general class of networks we consider, directed networks with arbitrary eigenvalues (and thereby arbitrary time constants), we suggest strategies based on a novel characterization of network non-normality as imbalance in the distribution of energy over the network. Our formulation allows to quantify network non-normality at a node level as combination of two different centrality metrics. The first measure quantifies the influence that each node has on the rest of the network, while the second measure instead describes the ability to control a node indirectly from the other nodes. Selecting the nodes that maximize the network non-normality as driver nodes significantly reduces the energy needed for control.

Growing a network, i.e. adding more edges to it, is a promising alternative to reduce the energy needed to control it. We approach this by deriving a sensitivity function that enables to quantify the impact of an edge modification with the $\mathcal{H}_{2}$ and $\mathcal{H}_{\infty}$ norms, which in turn can be used to design edge additions that improve commonly used control energy metrics. 



\section{Populärvetenskaplig sammanfattning}

Det reglerteoretiska begreppet styrbarhet avser förmågan att styra ett system till ett önskat tillstånd med ett lämpligt val av styrsignaler. Styrbarhet av komplexa nätverk såsom trafiknätverk, elnät, regulatoriska gennätverk m.m. kan exempelvis möjliggöra effektiv drift eller helt nya applikationer. Det finns dock flera utmaningar med att tillämpa reglerteori på komplexa nätverk, och den här avhandlingen handlar om några av dessa. Mer specifikt så undersöks hur ett givet nätverk kan göras styrbart till lägsta möjliga kostnad genom antingen placering av styrsignaler eller genom att utöka nätverket med fler anslutningar mellan dess noder. De kostnader som vi söker minimera är dels antalet styrsignaler, men också det arbete/den energi som de måste utöva.

En styrsignal kallas unilateral om den kan anta endast positiva eller endast negativa värden. Beaktat de många tillämpningsområden där sådana styrsignaler är vanliga, omformulerar vi klassiska styrbarhetsresultat för detta specialfall till en mer beräkningseffektiv form för analys i stor skala. Givet antagandet att varje styrsignal endast påverkar en enda nod direkt (samt indirekt dess grannar o.s.v.), visar vi att det unilaterala styrbarhetsproblemet i hög grad är strukturellt: utifrån nätverkets topologi härleds teoretiska gränser för det lägsta antal styrsignaler som krävs för styrbarhet, gränser liknande de som redan finns etablerade för antalet obegränsade styrsignaler, som kan anta både positiva och negativa värden. Med en algoritm för placering av unilaterala styrsignaler visar vi dessutom att de teoretiska gränserna ofta kan uppnås.

Ett nätverk kan vara styrbart i teorin men ändå inte i praktiken om till exempel orimliga energimängder krävs för att styra det i någon riktning. För fallet med styrsignaler som kan vara både positiva och negativa visar vi att energibehoven beror på tidskonstanterna i nätverkets moder. Med längre tidskonstanter behövs mindre energi. Vi föreslår också flera olika strategier för hur styrsignaler kan placeras för att minska energibehoven och därmed förbättra den "praktiska graden av styrbarhet”. I det mest generella fallet, ett riktat nätverk med godtyckliga egenvärden (och därmed godtyckliga tidskonstanter), föreslår vi strategier baserade på en karaktärisering av icke-normalitet hos nätverk som obalans i fördelningen av energi över nätverket. Med denna karaktärisering kan vi kvantifiera icke-normalitet hos varje enskild nod som en kombination av två olika centralitetsmått: det första måttet speglar nodens inflytande över resten av nätverket, och det andra, möjligheten att styra noden indirekt från andra noder. Genom att placera styrsignaler på de noder som maximerar nätverkets icke-normalitet så minskar mängden energi som behövs för styrning avsevärt.

Att utöka ett nätverk med fler anslutningar är ett alternativ till att placera ut fler styrsignaler som har stor potential att minska energin som behövs för att styra det. Vi tar oss ann detta genom att härleda en känslighetsfunktion som vi använder för att kvantifiera effekterna av att lägga till eller ta bort kopplingar i nätverket. Detta kan i sin tur användas för att utforma nya anslutningar som förbättrar nätverkets praktiska grad av styrbarhet. 



\section{Acknowledgments}

First of all, I would like to thank my supervisor Claudio Altafini for your guidance and encouragement. You have been a great source of ideas and inspiration, thank you for all the effort that you have invested!

When doing research, I have often found myself stuck with things that I thought I knew but suddenly started to doubt. In such situations my co-supervisor Anders Helmersson has been more than helpful with his broad and solid knowledge of control theory, thank you!

I would like to thank Svante Gunnarsson and Martin Enqvist, former resp. present head of the Division of Automatic Control at Linköping University. You have both contributed with great leadership! To spend my days reading, learning and developing myself, and even get decently paid for it, is truly a privilege. Also thank you Ninna Stensgård for kindly helping me with administrative tasks.

To all my dear colleagues at the automatic control group, thank you for contributing to a nice and friendly atmosphere. We have shared offices, conference trips, lunches, PhD courses, and many memorable conversations.

I would also like to take the opportunity to thank my family. My father Göte and brother Thomas, always supportive and kind. My mother Christina, often in my thoughts, was inspiring with her genuine interest in just about everything. My wife Jenny, thank you for your patient and loving support. You and our children are what's truly important to me. 



\section{Contents}

\section{Background}

1 Introduction 3

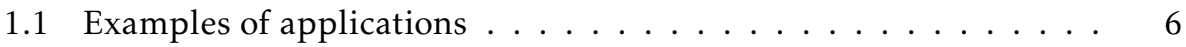

1.2 Contributions ....................... 8

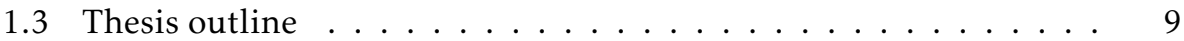

2 Graphs and networks 13

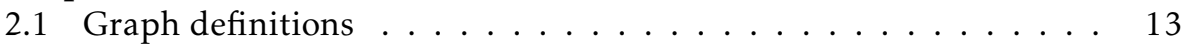

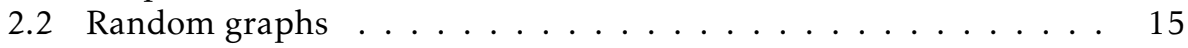

2.3 Network centrality metrics . . . . . . . . . . . . 16

3 Controllability 19

3.1 Controllability of LTI systems . . . . . . . . . . . . . . . . . . . 19

3.2 Controllability with constrained inputs ........... 22

3.3 Structural controllability . . . . . . . . . . . . 23

3.4 Minimal controllability of complex networks . . . . . . . . . 25

3.5 Minimum energy control of complex networks . . . . . . . 26

4 Norms of systems: $\mathcal{H}_{2}$ and $\mathcal{H}_{\infty}$

5 Concluding remarks $\quad 31$

$\begin{array}{ll}\text { Bibliography } & 35\end{array}$

\section{Publications}

A Controllability of complex networks with unilateral inputs 41

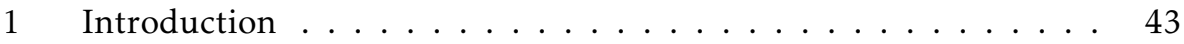

2 Methods ........................ 46

3 Results ...................... 54

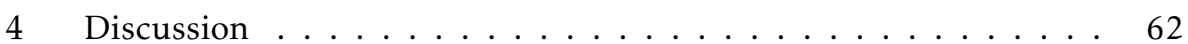


A Positive linear dependence . . . . . . . . . . . . . . . . 64

B Derivation of conditions for unilateral controllability . . . . . . 65

C Algorithm for construction of unilateral control inputs . . . . . . 66

C.1 Greedy input selection with simple real eigenvectors . . . . 67

C.2 Extension for multidimensional real eigenspaces . . . . . 68

D Derivation of bound on the minimum number of unilateral controls 69

D.1 Structure of the left null space of A . . . . . . . . . 69

D.2 Positive spanning with structurally disjoint vectors . . . . 70

E Derivations of results for specific network structures . . . . . . 72

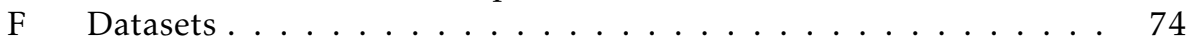

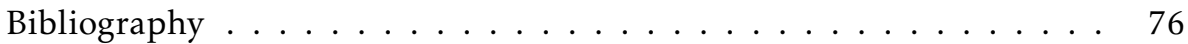

B Minimum energy control for complex networks 81

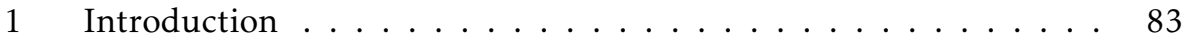

2 Methods ....................... 85

3 Results ..................... 88

4 Discussion ........................ 106

A Control energy formulations . . . . . . . . . . . . 109

A.1 Finite time horizon . . . . . . . . . . . . . . . 109

A.2 Infinite time horizon . . . . . . . . . . 110

A.3 Mixed Gramian in infinite and finite time horizon . . . . 112

B Control of coupled harmonic oscillators . . . . . . . . 112

C Controllability with bounded controls . . . . . . . . . 117

D Datasets . . . . . . . . . . . . . . . . . . 117

Bibliography ...................... 123

C Centrality measures and the role of non-normality for network con$\begin{array}{ll}\text { trol energy reduction } & 127\end{array}$

1 Introduction . . . . . . . . . . . . . . . . . . . . . . . 129

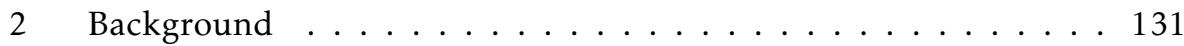

2.1 Notation . . . . . . . . . . . . . . . . . . 131

2.2 Controllability . . . . . . . . . . . . . . . . 131

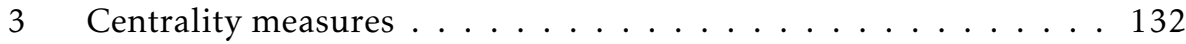

3.1 Walk energy . . . . . . . . . . . . . . . . 133

3.2 Node-to-network centrality . . . . . . . . . . . . . . 134

3.3 Network-to-node centrality . . . . . . . . . . . 135

3.4 Interpretation of the centrality measures as $\mathcal{H}_{2}$ system norms 138

4 Non-normality and balanced systems . . . . . . . . . . . . . . . 139

4.1 Characterization of network non-normality . . . . . . 139

4.2 Non-normality in a node . . . . . . . . . . . . . 140

4.3 Non-normality in a set of nodes . . . . . . . . . . . 141

5 Driver node placement . . . . . . . . . . . . . . . 142

5.1 Simulations . . . . . . . . . . . . . . . . . 143

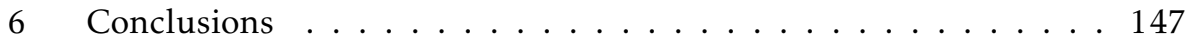

A Networks with discrete time dynamics . . . . . . . . . . . 148

Bibliography . . . . . . . . . . . . . . . 150 
D On the impact of edge modifications for networked control systems 153

1 Introduction . . . . . . . . . . . . . . . 155

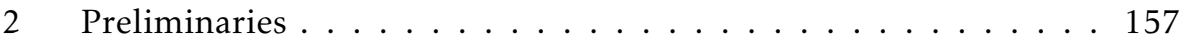

2.1 Notation . . . . . . . . . . . . . . . 157

2.2 Network model . . . . . . . . . . . . . . . 158

2.3 System norms and network centrality measures . . . . . . 159

3 The Delta System . . . . . . . . . . . . . . . . . 161

4 Large scale analysis over all edges . . . . . . . . . . . . . . 163

4.1 Stability bounds and the $\mathcal{H}_{\infty}$ norm of $G^{\delta} \ldots \ldots 163$

4.2 The $\mathcal{H}_{2}$ norm of $G^{\delta} \ldots \ldots \ldots \ldots 616 \ldots$

4.3 Interpretations . . . . . . . . . . . 168

5 Applications .......................... 169

5.1 Edge modifications and the degree of controllability . . . 169

5.2 Network fragility ... . . . . . . . . . . 169

5.3 Numerical results . . . . . . . . . . . . . . 170

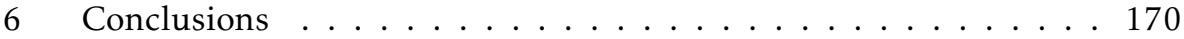

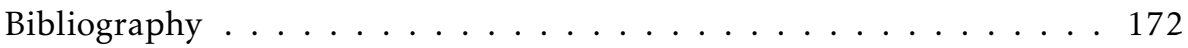


Part I

Background 



\section{1}

\section{Introduction}

Large scale, complex networks appear in a broad spectrum of scientific disciplines, ranging from Biology to Social Sciences, from Technology to Engineering. In this thesis we consider the problem of controlling them. While control methods have been developed primarily to deal with man-made technical systems, such as cars, aircraft etc., in many of the contexts in which complex networks are investigated the networks do not represent engineering systems but rather large assemblies of entities (nodes) having some form of interactions represented as the edges of a graph. In this perspective, the control of a network poses challenges which cannot always be answered by classical control theory.

Regardless of the specific applicative context, we will always assume that to the $n$ nodes of the graph is associated a vector of finite size with state variables, $x(t) \in \mathbb{R}^{n}$. Controlling a complex network means steering its state variables using the available control inputs. For example, the vector element $x_{i}(t)$ can denote the amount of traffic that passes through node $i$ in a road network, and a control objective could be to avoid congestion. In a food web instead, the state $x_{i}(t)$ might denote the population of a species and a control objective to preserve biodiversity. Depending on the context, many are the possible ways to define control inputs on networks, from traffic lights in traffic networks (Tewolde, 2012) to drugs in biological networks (Torres and Altafini, 2016), from dams in irrigation networks (Mareels et al., 2005) to opinion makers in social networks, etc.

Controlling a network does not require to have control authority on each and every node. If the graph of the network is available, then interactions among nodes can be used to forward the effect of a control input, i.e. a few nodes, called driver nodes, on which external control inputs are acting can be used to indirectly control also the other nodes over the edges of the network. Much of the research in the field concerns how control inputs should be placed in order to render a given network controllable at a minimum cost. If we take as cost function the 
number of control inputs needed to achieve controllability, then finding a minimal set of driver nodes is a problem of interest. If instead the cost is associated to the energy that the driver nodes must exert in order to steer the state of the network in an arbitrary direction, then another set of driver nodes might be a better choice. In fact, a network that is controllable in a control theoretic sense is often not controllable in practice. It could for instance be the case that completely unreasonable amounts of control energy are required to steer the network in some directions. In the context of large-scale networks, the need to achieve a "practical degree of controllability" is much more pressing than in classical (small-scale) control systems (Bof et al., 2017; Chen et al., 2016; Li et al., 2016; Nacher and Akutsu, 2014; Olshevsky, 2016; Pasqualetti et al., 2014; Summers et al., 2016; Tzoumas et al., 2016; Yan et al., 2012, 2015). Besides driver node placement, another method to achieve controllability at minimum cost is to grow the network by adding more edges to it. This approach has however received significantly less attention by the research community.

The dynamics of the networks are normally expected to be nonlinear, but poor knowledge of the precise dynamics and the mere complexity of the system make any substantial overarching conclusion difficult to reach without simplifications. For a large scale analysis, a usual starting point in the literature on control of complex networks is instead a Linear Time-Invariant (LTI) network model,

$$
\dot{x}(t)=A x(t)+B u(t) .
$$

The matrix $A \in \mathbb{R}^{n \times n}$, normally sparse, describes the interactions between the entities of the network, and the matrix $B \in \mathbb{R}^{n \times m}$ how the control inputs $u(t)=$ $\left[u_{1}(t) \ldots u_{m}(t)\right]^{\top}$ enter into the system. The natural mathematical representation of a network is however a graph, and the network model (1.1) corresponds to the directed graph $\mathcal{G}(A)$ with the set of nodes $\mathcal{V}=\left\{v_{1}, \ldots, v_{n}\right\}$ and edges $\mathcal{E}=$ $\left\{\left(v_{i}, v_{j}\right), i, j\right.$ s.t. $\left.A_{j i} \neq 0\right\}$ with weights given by the numerical values of the elements in $A$. A common assumption is that $B=\left[e_{k_{1}} \ldots e_{k_{m}}\right]$ where $e_{k}, k \in 1, \ldots, n$, is the $k$-th vector of the canonical basis of $\mathbb{R}^{n}$, which corresponds to placing each control input on a single node of the network (the nodes $v_{k_{1}}, \ldots, v_{k_{m}}$ are the driver nodes). Both representations, linear system of ODEs and graphical, are useful and often complement each other.

A topological, or structural, network model is represented by a graph without edge weights. Such model can be used when the interactions between the network components are known but limited or no information is available about their strengths and functional form. Algebraically, this can be represented as each entry of the adjacency matrix $A$ being zero or non-zero. Under some assumptions on the values of the non-zero parameters, this simple model can be used to determine if a system is controllable or not. Such notion is referred to as structural controllability (Lin, 1974) and it is based on the fact that controllability with unrestricted control inputs is a generic property that holds for almost all parameter values. Of course, when the edge weights are known, then a more thorough analysis can be made, which can help in quantifying the practical degree of controllability. 

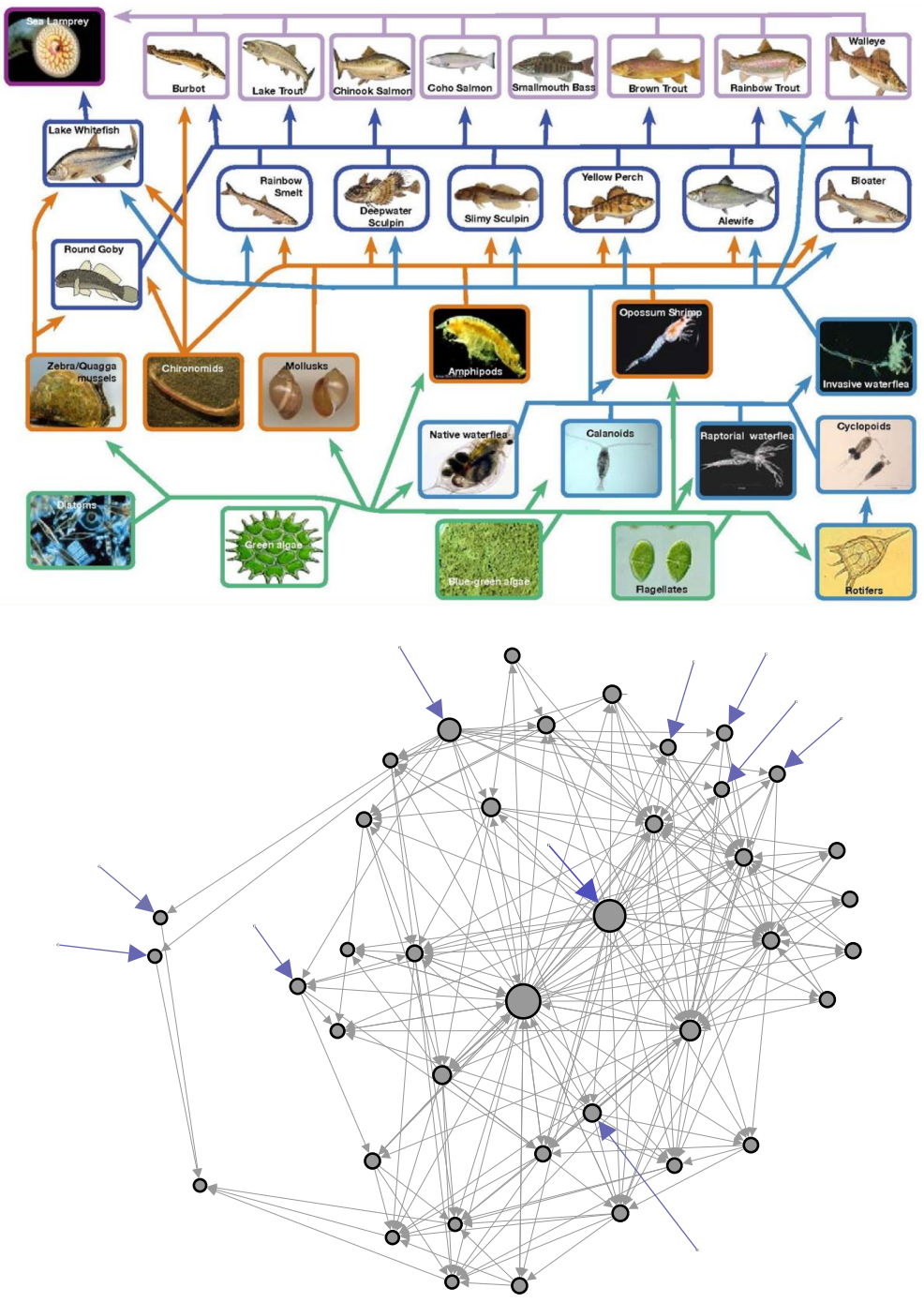

Figure 1.1: Lake Michigan food web (Madenjian et al., 2002). The graph represents a dynamical model in which each state is the population of a species. A minimal set of control inputs that render the network controllable is indicated with blue arrows. For instance feeding and breeding, hunting and fishing are control inputs in an ecosystem with the special property that they assume either positive or negative values but not both. 


\subsection{Examples of applications}

Intelligent transportation systems (ITS) are technologies characterized by information, dynamic feedback and automation that address various transportation problems. Perhaps the most important problems are caused by traffic congestion resulting in enormous environmental and economic costs and waste of time. The basis for ITS is the collection and processing of information from sensors integrated in the traffic infrastructure, sensors in the vehicles, satellite information, maps etc. A central system that collects information from different sources can monitor the state of the traffic network, enabling network level traffic control to avoid congestion and achieve optimal utilization of roads and other resources. The actual control inputs can be for instance variable speed limits, control of traffic lights or toll fees. Furthermore, vehicles with telematics already receive information that they benefit from, e.g. real-time route guidance, accident alert systems etc. In a near future with autonomous cars the possibilities for network level traffic control will increase even more.

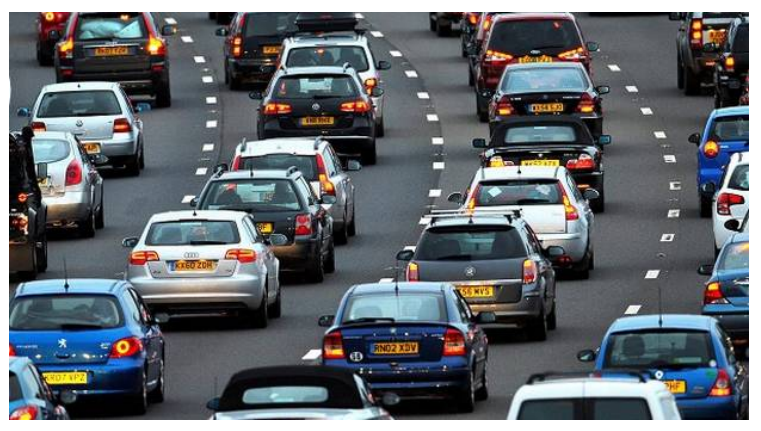

Figure 1.2: New technologies for dynamic traffic network control can reduce the economical and environmental costs of congestion.

The smart power grid refers to technologies that allows for communication between the grid components, sensing along the transmission lines and automation and control for its function. The power grid is a network of generators, transmission lines, substations, transformers, consumers etc. with the task to produce and distribute as much power as is needed. If not successful, the grid voltage could drop, causing the grid to become unstable and in worst case lead to power outage. Traditionally, grid operators have limited information about how the power is flowing through the grid, but with smart grid technologies the state of the network can be monitored and proper control actions taken for its function, leading to increased reliability, availability and efficiency. 
One upcoming challenge for the power grid function is the integration of variable sources of power such as wind power and solar power, which supply an increasing fraction of power to the grid. Since their outputs cannot be controlled there must be other mechanisms for controlling the power supply and demand in the network. One way to do that is to balance the outputs of the variable power sources with other power sources and another is to add energy storage capabilities to the network. Control inputs can also be financial incentives, e.g. variable pricing, for consumers to shift their power demand to off-peak hours. Even rewiring the grid (i.e. adding edges) can be a form of control.

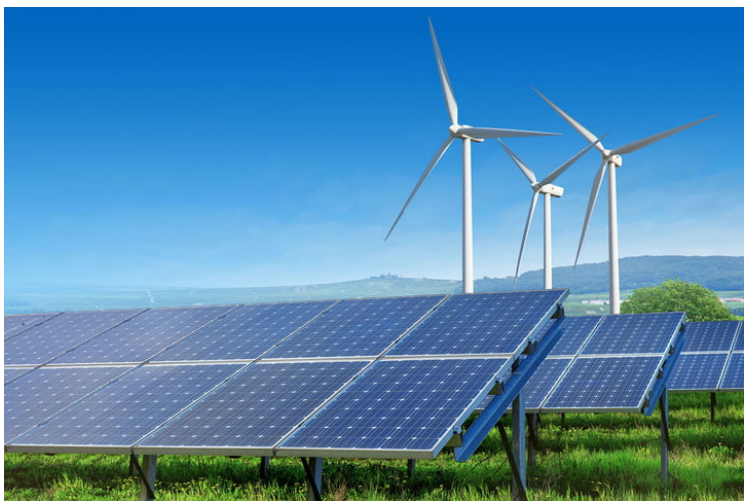

Figure 1.3: The outputs of wind and solar power cannot be controlled, hence a smart grid needs other control mechanisms for balancing the power supply and demand as these power sources become increasingly important. 


\subsection{Contributions}

Although the different articles in this thesis consider different network models and problem formulations, the overall theme for all of them is investigating how the problem of controlling complex networks can be made more realistic. For instance, in Paper A we restrict the LTI model (1.1) and consider unilateral control inputs, i.e. inputs that can assume either positive or negative values but not both. In many different fields in which controllability of large scale networks is studied, the control action is intrinsically constrained, and this is perhaps the most common form of constraint. In Papers B and C we investigate the practical degree of controllability of networks based on energy considerations, and propose methods for driver node placement that significantly reduce the amount of control energy needed to steer the network in different directions. Although the methods of e.g. Paper C stem from a theoretical characterization of network non-normality, are they very practical in their implementation. Paper D points to edge additions as an alternative approach to tackle network controllability problems. Also in this paper the focus is on providing practical tools for large scale analysis of complex networks.

The main contributions of the thesis are detailed below.

- Exact theoretical bounds on the minimal number of controls required for unilateral controllability of a given network are derived from the network topology. The bounds can often be achieved by an algorithm developed to identify a near minimal set of unilateral control inputs. (Paper A)

- We show how the energy required for controlling a network depends on the time constants of the modes of the network. (Paper B)

- Two centrality measures quantifying the importance of the different nodes in controlling a network are proposed. Driver node placement based on combinations of these measures results in reduced energy requirements for controlling a network. (Papers B and C)

- An equivalence between a network with normal adjacency matrix and a system with balanced realization is established. For networks with nonnormal adjacency matrix, we quantify the network non-normality by the imbalance in distribution of energy over the network. (Paper C)

- For a given network with input and output nodes, a transfer function formulation for the changes in output caused by edge-modifications is derived. The formulation can be used to design edge modifications for controllability in a large scale setting. (Paper D) 


\subsection{Thesis outline}

The thesis is divided into two parts, with background material in Part I and edited versions of published papers in Part II.

\section{Part I - Background}

The first part introduces the theoretical background for the publications in Part II. Chapter 2 presents basic concepts from graph theory and discusses the use of random networks and network centrality metrics for the development of algorithms. Chapter 3 provides background theory on controllability, with focus on notions of controllability that have been recently adapted to the context of complex networks.

\section{Part II - Publications}

The second part consists of edited versions of four publications. Below is a summary of each paper.

\section{Paper A: Controllability of complex networks with unilateral inputs}

Paper $\mathrm{A}$ is an edited version of

Gustav Lindmark and Claudio Altafini. Controllability of complex networks with unilateral inputs. Scientific Reports, 7:1824, 2017a. doi: 10.1038/s41598-017-01846-6.

Summary: In Paper A we study the problem of controlling complex networks with unilateral controls. Given a complex network represented by the adjacency matrix $A$, an algorithm is developed that constructs an input matrix $B$ such that the resulting system $(A, B)$ is controllable with a near minimal number of unilateral control inputs. This is made possible by a reformulation of classical conditions for controllability that casts the minimal unilateral input selection problem into well known optimization problems. We identify network properties that make unilateral controllability relatively easy to achieve as compared to unrestricted controllability. The analysis of the network topology for instance allows us to establish theoretical bounds on the minimal number of controls required. For various categories of random networks as well as for a number of real-world networks these lower bounds are often achieved by our heuristics.

Contribution and background: The author of this thesis contributed with the majority of the work including theoretical derivations, implementations, numerical calculations and the written manuscript. 


\section{Paper B: Minimum energy control for complex networks}

Paper B is an edited version of

Gustav Lindmark and Claudio Altafini. Minimum energy control for complex networks. Scientific Reports, 8(1):3188, 2018.

Summary: The aim of Paper B is to shed light on the problem of controlling a complex network with minimal control energy. We show first that the control energy depends on the time constants of the modes of the network, and that the closer the eigenvalues are to the imaginary axis of the complex plane, the less energy is required for complete controllability. In the limit case of networks having all purely imaginary eigenvalues (e.g. networks of coupled harmonic oscillators), several constructive algorithms for minimum control energy driver node selection are developed. A general heuristic principle valid for any directed network is also proposed: the overall cost of controlling a network is reduced when the controls are concentrated on the nodes with highest ratio of weighted outdegree vs. indegree.

Contribution and background: The author of this thesis contributed with implementations, analysis and reviewing the manuscript.

\section{Paper C: Centrality measures and the role of non-normality for network control energy reduction}

Paper $\mathrm{C}$ is an edited version of

Gustav Lindmark and Claudio Altafini. Centrality measures and the role of non-normality for network control energy reduction. Preprint, $2020 b$.

Summary: In this paper, the empirical results of Paper B are put into a more solid formal basis and interpreted in terms of the algebraic properties of the adjacency matrix of the network. Combinations of Gramian-based centrality measures are used for driver node selection in complex networks in order to simultaneously take into account conflicting control energy requirements, like minimizing the average energy needed to steer the state in any direction and the energy needed for the worst direction. The selection strategies that we propose are based on a novel characterization of the network non-normality, a concept we show is related to the idea of balanced realization.

Contribution and background: The author of this thesis contributed with the majority of the work including theoretical derivations, implementations, numerical calculations and the written manuscript. 


\section{Paper D: On the impact of edge modifications for networked control systems}

Paper D is an edited version of

Gustav Lindmark and Claudio Altafini. On the impact of edge modifications for networked control systems. In Proceedings of the 2020 IFAC World Congress, to appear, 2020a.

Summary: In complex networks, the addition/removal of edges can occur both naturally and by design. This paper investigates the impact of such changes on the system properties of the network. Growing a network by the addition of edges has for instance been suggested as a way to improve network robustness to external disturbances. Moreover, when network controllability is considered, designing edge additions is a promising alternative to add more actuation capabilities in order to improve different performance metrics. We quantify the impact of an edge modification with the $\mathcal{H}_{\infty}$ and $\mathcal{H}_{2}$ norms. For networks with positive edge weights we show how the $\mathcal{H}_{\infty}$ norm can be computed exactly for each possible single edge modification, while for the $\mathcal{H}_{2}$ norm we instead obtain a lower bound. This bound is linked to the trace of the controllability Gramian, a commonly used metric for the control energy, hence it can be used to reduce the energy needed for control. However, when edge additions create new cycles in the network, this may lead to instability. For positive networks we develop criteria to identify when this happens.

Contribution and background: The author of this thesis contributed with the majority of the work including theoretical derivations, implementations, numerical calculations and the written manuscript.

\section{Publications}

Works published by the author of this thesis are listed below in chronological order. Publications indicated by a $\star$ are included in Part II of this thesis.

Gustav Lindmark and Claudio Altafini. Positive controllability of large-scale networks. In Proceedings of the 2016 European Control Conference (ECC), pages 819-824. IEEE, 2016.

Francesca Ceragioli, Gustav Lindmark, Clas Veibäck, Niklas Wahlström, Martin Lindfors, and Claudio Altafini. A bounded confidence model that preserves the signs of the opinions. In Proceedings of the 2016 European Control Conference (ECC), pages 543-548. IEEE, 2016.

Gustav Lindmark and Claudio Altafini. Topological aspects of controlling large scale networks with unilateral inputs. In Proceedings of the 2017 IFAC World Congress, volume 50, pages 8315-8320. Elsevier, 2017 d. 
Gustav Lindmark and Claudio Altafini. Minimum energy control for networks of coupled harmonic oscillators. In Proceedings of the 2017 IFAC World Congress, volume 50, pages 8321-8326. Elsevier, 2017c.

Gustav Lindmark and Claudio Altafini. A driver node selection strategy for minimizing the control energy in complex networks. In Proceedings of the 2017 IFAC World Congress, volume 50, pages 83098314. Elsevier, 2017b.

$\star$ Gustav Lindmark and Claudio Altafini. Controllability of complex networks with unilateral inputs. Scientific Reports, 7:1824, 2017a. doi: 10.1038/s41598-017-01846-6.

$\star$ Gustav Lindmark and Claudio Altafini. Minimum energy control for complex networks. Scientific Reports, 8(1):3188, 2018.

Gustav Lindmark and Claudio Altafini. Combining centrality measures for control energy reduction in network controllability problems. In 2019 European Control Conference (ECC), pages 1518-1523. IEEE, 2019.

$\star$ Gustav Lindmark and Claudio Altafini. On the impact of edge modifications for networked control systems. In Proceedings of the 2020 IFAC World Congress, to appear, 2020a.

$\star$ Gustav Lindmark and Claudio Altafini. Centrality measures and the role of non-normality for network control energy reduction. Preprint, 2020b. 


\section{2}

\section{Graphs and networks}

A natural and very useful mathematical representation of a network is a graph, hence in this chapter we present basic concepts from graph theory. We also discuss the use of random networks and network centrality metrics for the development of algorithms.

\subsection{Graph definitions}

A graph $\mathcal{G}$ is a pair $\mathcal{G}=(\mathcal{V}, \mathcal{E})$ where $\mathcal{V}=\left\{v_{1}, \ldots, v_{n}\right\}$ is a finite set of nodes (or vertices $)$ and $\mathcal{E}$ is a set of edges. An edge $\left(v_{i}, v_{j}\right) \in \mathcal{E}$ connects the nodes $v_{i}, v_{j} \in \mathcal{V}$, and the nodes are said to be adjacent to each other and incident to the edge $\left(v_{i}, v_{j}\right)$. In a directed graph, or digraph, the edge $\left(v_{i}, v_{j}\right)$ is directed from $v_{i}$ to $v_{j}$, while in an undirected graph, $\left(v_{i}, v_{j}\right) \in \mathcal{E} \Rightarrow\left(v_{j}, v_{i}\right) \in \mathcal{E}$. A weighted graph has weights associated with each edge.

We say that $\mathcal{G}^{*}=\left(\mathcal{V}^{*}, \mathcal{E}^{*}\right)$ is a subgraph of $\mathcal{G}=(\mathcal{V}, \mathcal{E})$ if $\mathcal{V}^{*} \subset \mathcal{V}, \mathcal{E}^{*} \subset \mathcal{E}$ and the edges in $\mathcal{E}^{*}$ only connect nodes in $\mathcal{V}^{*}$. A path $\mathcal{P}$ in $\mathcal{G}$ is a subgraph of the form $\mathcal{V}^{*}=\left\{v_{i_{1}}, \ldots, v_{i_{j}}\right\}$ and with the edges $\mathcal{E}^{*}=\left\{\left(v_{i_{1}}, v_{i_{2}}\right), \ldots,\left(v_{i_{j-1}}, v_{i_{j}}\right)\right\}$. The path is directed from $v_{i_{1}}$ to $v_{i_{j}}$ and the number of edges is the path length. A cycle is a path in which $v_{i_{1}}=v_{i_{j}}$. The simplest form of cycle is a node $v_{i}$ with a self-loop, i.e. an edge $\left(v_{i}, v_{i}\right)$.

A graph can be specified by its adjacency matrix $A$, i.e. $\mathcal{G}=\mathcal{G}(A)=(\mathcal{V}, \mathcal{E})$. With $|\mathcal{V}|=n$, the matrix $A$ is $n \times n$. When the graph is unweighted, $A_{j i}=1$ if $\left(v_{i}, v_{j}\right) \in \mathcal{E}$ and $A_{j i}=0$ if $\left(v_{i}, v_{j}\right) \notin \mathcal{E}$. In a weighted graph the element $A_{j i}$ specifies the weight of the edge $\left(v_{i}, v_{j}\right)$, and $A_{j i} \neq 0$ if $\left(v_{i}, v_{j}\right) \in \mathcal{E}$.

An undirected graph is connected if there is a path between any two nodes in the graph. The connected components of a graph are its maximal connected subgraphs. Analogously, a directed graph is strongly connected if there is a directed path from each node to every other node, and the strongly connected components 
are its maximal strongly connected subgraphs. The minimal connected/strongly connected component is a single node.

Two distinct nodes that are adjacent (i.e. connected by an edge) are neighbors. For undirected graphs, the degree of a node is its number of incident edges. The degree distribution of a graph is the distribution of the degrees of its nodes. In a directed graph, the in-neighbors of the node $v_{i}$ is given by the set of nodes $\left\{v_{j}, j\right.$ s.t. $\left.\left(v_{j}, v_{i}\right) \in \mathcal{E}\right\}$, and the out-neighbors by the set $\left\{v_{k}\right.$, k s.t. $\left.\left(v_{i}, v_{k}\right) \in \mathcal{E}\right\}$. The indegree of a node is the number of edges incoming into it, and the outdegree the number of outgoing edges from it. The definitions of indegree and outdegree distributions of a directed graph follow. We say that a node in a directed network is a root if it has indegree zero, and a leaf if it has outdegree zero.

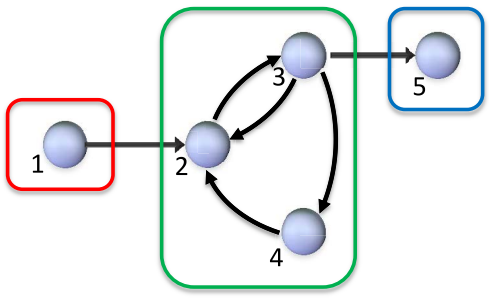

(a)

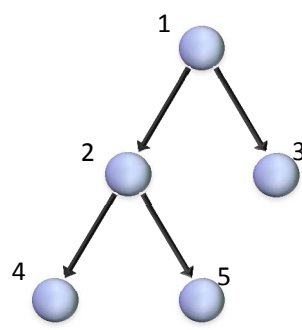

(b)

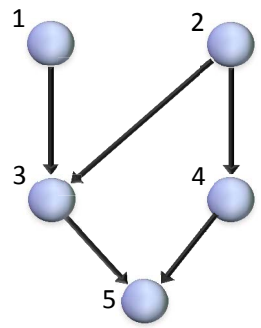

(c)

Figure 2.1: Examples to illustrate key-concepts from graph theory. (a): The graph has three strongly connected components marked in red/green/blue. The node $v_{1}$ is a root and $v_{5}$ a leaf. The strongly connected component marked in green contains the cycle $\left\{v_{2}, v_{3}, v_{4}, v_{2}\right\}$. In the graph, $v_{2}$ is the node with highest in-degree $\left(k_{\text {in }}=3\right)$ and $v_{3}$ has the highest out-degree $\left(k_{\text {out }}=3\right)$. (b): Rooted directed tree. (c): Directed acyclic graph.

\section{Some special classes of graphs}

A tree is an undirected graph where any two nodes are connected with exactly one path, hence a tree is acyclic. In a directed rooted tree one node is the root and all edges are pointing either away from or towards the root such that there is exactly one path from the root to each other node or vice versa. Directed rooted trees belong to the important class of graphs Directed acyclic graphs, DAGs. As the name suggests, a DAG is directed and contain no cycles. In a DAG, there is a topological ordering of the nodes such that $i<j$ for all $\left(v_{i}, v_{j}\right) \in \mathcal{E}$. The topological ordering is unique if and only if there is a directed path containing all the nodes. DAGs are used for instance in computer science to identify dependencies between the execution of different tasks and detect deadlocks, or to model probabilities and causality. The adjacency matrix of a DAG without self-loops is nilpotent.

A graph is said to be complete if there are edges between all pairs of nodes. If the graph is directed then there must be edges in both directions. All off-diagonal entries of the corresponding adjacency matrix are non-zero (the diagonal entries 
are also non-zero if there are self-loops).

\subsection{Random graphs}

It is often interesting to generate random graphs, for instance to evaluate different graph methods or algorithms. By applying a method on a large number of random graphs, statistically sound conclusions can be drawn about its performances. A random graph is described either by a probability function or by a process that generates it. Different classes of random graphs have different characteristics. One property that often is of interest is the degree of clustering. Clustering is when the neighbors of a node tend to be also neighbors to each other. Another important graph property is the connectivity. A graph with high connectivity is connected and remains connected even when several nodes or edges are removed from the graph.

The generation of random graphs with specific properties is an active area of research. However, for this thesis it is enough to present the two perhaps most common classes of random graphs, Erdős-Rényi networks and scale-free networks. (The terms graph and network are synonymous in this context.)

\section{Erdős-Rényi networks}

In the Erdős-Rényi random network model, the probability that an edge connecting $v_{i}$ and $v_{j}$ exists is $p$ for all $v_{i}, v_{j} \in \mathcal{V}$. That is, the probability $p$ is independent of what other edges there are and equal for all pairs of nodes. Denote by $P(k)$ the probability that a node has degree $k$. In an Erdős-Rényi network with $n$ nodes,

$$
P(k)=\left(\begin{array}{c}
n-1 \\
k
\end{array}\right) p^{k}(1-p)^{n-1-k}, k=0,1, \ldots, n,
$$

i.e. the function $P(k)$ is a binomial probability distribution with expected value $n p$ (the nodes have on average $n p$ neighbors). Moreover, the probabilistic degree distribution of Erdős-Rényi networks becomes $n P(k)$. Erdős-Rényi networks are characterized by low clustering but high connectivity (Newman, 2010).

\section{Scale-free networks}

Scale-free networks are networks in which the degree distribution follows a power law,

$$
P(k)=C k^{-\gamma}, k=1,2, \ldots, n .
$$

Here, $C$ is a constant and $\gamma$ is the degree exponent of the power law. Several real world networks are claimed to be close to scale-free with $\gamma \in[2,3]$ (Barabási and Albert, 1999). That includes computer networks like the World Wide Web as well as collaboration networks. A Scale-free network is the result of a preferential attachment process in which the network is constructed incrementally by the 
addition of new nodes and edges. When an edge is added, the probability that it becomes incident to the existing node $v_{i}$ is higher the higher the degree of $v_{i}$ is. Hence, when new nodes are added, they are more likely to connect to already "important" nodes. Preferential attachment has been used to model several real world phenomena (Newman, 2010).

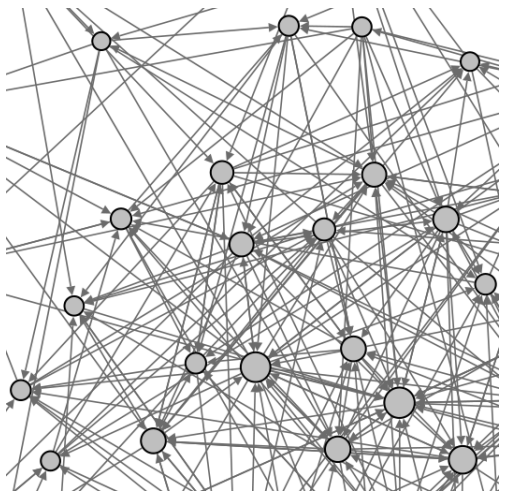

(a)

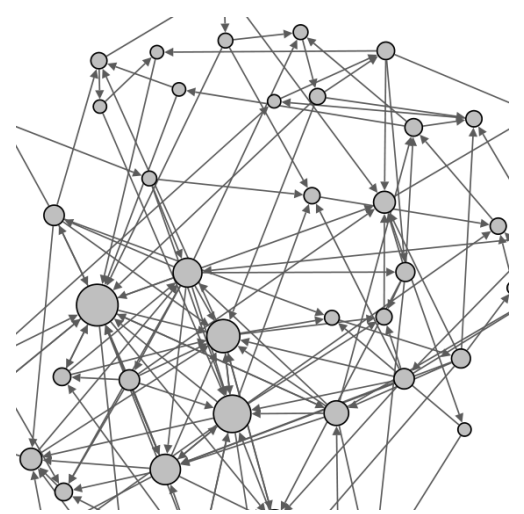

(b)

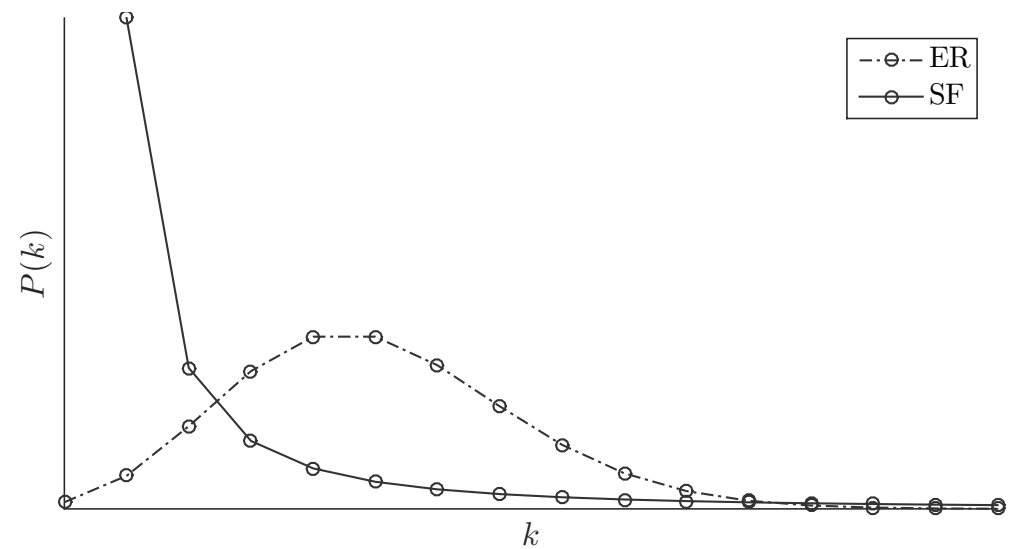

(c)

Figure 2.2: (a): Directed Erdős-Rényi network. (b): Directed scale free network. In scale-free networks a large fraction of the edges are concentrated around a few hubs while the large majority of the nodes have low degree. (c): The probability distributions $P(k)$ for Erdős-Rényi and scale-free networks.

\subsection{Network centrality metrics}

A node centrality metric quantifies how important, or central, the different nodes of a network are. There are numerous different centrality metrics and their relevance depends on the type of network and the context in which they are used, 
see for instance Newman (2010) for an overview. The simplest centrality metric is probably the degree centrality, which is just the degree of the different nodes. It can for instance be a measure of how influential a person is in a social network or used to study the spread of infections in epidemics. The eigenvector centrality of a node differs from the degree centrality in that the different connections of the node are not equally important. Instead, the centrality of a node is higher if its neighbors have high centrality themselves. Other examples of network centrality metrics are for instance PageRank, known for being used by Google Search to rank websites, and the hubness/authority measures used for instance in the HITS algorithm. In particular, in a directed network the hub centrality is related to the outgoing edges while the authority centrality is related to the incoming edges.

One way to approach the problem of driver node placement for control of complex networks is to quantify the importance of the different nodes with network centrality measures. There are several centrality metrics proposed for control, see for instance Bof et al. (2017); Liu et al. (2012); Pasqualetti et al. (2014). In paper $\mathrm{C}$ we propose driver node placement based on the combination of two different network centrality metrics. 



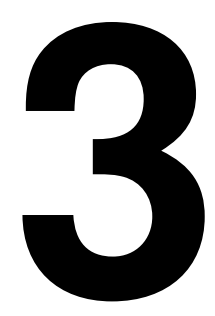

\section{Controllability}

This chapter provides background theory on controllability that the publications in Part II of the thesis are based on. The concept of controllability has a long history, it was first introduced in Kalman (1963) and has played a fundamental role in system theory since then. While the concept includes a large body of theory and many ramifications (Rugh, 1996; Sontag, 2013), this presentation focuses on notions of controllability that have been recently adapted to the context of complex networks. This includes controllability of Linear Time-Invariant (LTI) systems, systems with linear dynamics but constrained control inputs, structural controllability and interpretations of the controllability and reachability Gramians.

\subsection{Controllability of LTI systems}

Consider a continuous-time LTI system

$$
\dot{x}=A x+B u
$$

where $x \in \mathbb{R}^{n}$ is the state vector, $A \in \mathbb{R}^{n \times n}$ is the state update matrix, $B \in \mathbb{R}^{n \times m}$ is the input matrix, and $u$ is the $m$-dimensional input vector. Given the initial state $x(0)=x_{0}$, the solution to the differential equation (3.1) is

$$
x(t)=e^{A t} x_{o}+\int_{0}^{t} e^{A(t-\tau)} B u(\tau) d \tau .
$$

Definition 3.1 (Controllability). The linear state equation (3.1) is called controllable (to the origin) on $\left[0, t_{f}\right]$ if given any initial state $x(0)=x_{o} \in \mathbb{R}^{n}$ there exists a continuous input signal $u(t)$ such that the corresponding solution of (3.1) satisfies $x\left(t_{f}\right)=0$ (Rugh, 1996). 
Closely linked to controllability (to the origin) is the term reachability (or controllability from the origin).

Definition 3.2 (Reachability). The system (3.1) is reachable (or controllable from the origin) on $\left[0, t_{f}\right]$ if for $x(0)=0$ and any given $x_{f} \in \mathbb{R}^{n}$ there exists a continuous input signal $u(t)$ such that the corresponding solution of (3.1) satisfies $x\left(t_{f}\right)=x_{f}$ (Rugh, 1996).

For continuous-time LTI systems the controllability and reachability properties are equivalent (Rugh, 1996). Moreover, if the control inputs are unconstrained, then a system that is controllable/reachable on $\left[0, t_{f}\right]$ is controllable/reachable on any non-empty time window.

The assumption that the LTI system (3.1) is controllable (hence also reachable) on $\left[0, t_{f}\right]$ is equivalent to (Zhou et al., 1996)

i) The controllability Gramian

$$
W_{c}\left(t_{f}\right)=\int_{0}^{t_{f}} e^{-A t} B B^{\top} e^{-A^{\top} t} d t
$$

is positive definite.

ii) The reachability Gramian

$$
W_{r}\left(t_{f}\right)=\int_{0}^{t_{f}} e^{A t} B B^{\top} e^{A^{\top} t} d t
$$

is positive definite.

iii) The controllability matrix

$$
\mathcal{C}=\left[B A B \ldots A^{n-1} B\right]
$$

has full row rank.

iv) The matrix $[A-\lambda I B]$ has full row rank for all $\lambda \in \mathbb{C}$.

v) Let $\lambda$ and $x$ be any eigenvalue and any corresponding left eigenvector of $A$, i.e., $x^{\mathrm{H}} A=x^{\mathrm{H}} \lambda$, then $x^{\mathrm{H}} B \neq 0$. Here $x^{\mathrm{H}}$ denotes the complex conjugate transpose of $x$.

vi) The eigenvalues of $A+B F$ can be freely assigned (with the restriction that complex eigenvalues are in conjugate pairs) by a suitable choice of $F$.

Condition iii) is often referred to as the Kalman rank condition, while the conditions $i v$ ) and $v$ ) are often called the Popov-Belevitch-Hautus ( $\mathrm{PBH})$ tests. The result $v i$ ) indicate why the concept of controllability plays a such important role 
in the classical control theory; the poles of a controllable system can be arbitrarily assigned with state feedback control. The controllability Gramian and the reachability Gramian are related by

$$
W_{r}\left(t_{f}\right)=e^{A t_{f}} W_{c}\left(t_{f}\right) e^{A^{\top} t_{f}} .
$$

In a controllable system, the state transfer from any $x_{o}$ into any other $x_{f}$ in time $t_{f}$ can be accomplished by many control inputs. In order to quantify how costly a state transfer is on a system, one can choose to consider the control input that minimizes the input energy, i.e., the functional

$$
\mathcal{E}\left(t_{f}\right)=\int_{0}^{t_{f}}\|u(\tau)\|^{2} d \tau .
$$

Such control can be computed explicitly (Antsaklis and Michel, 2005) as

$$
u^{*}(t)=B^{\top} e^{A^{\top}\left(t_{f}-t\right)} W_{r}^{-1}\left(t_{f}\right)\left(x_{f}-e^{A t_{f}} x_{o}\right), \quad t \in\left[0, t_{f}\right]
$$

and the corresponding transfer cost as

$$
\begin{aligned}
\mathcal{E}\left(t_{f}\right) & =\left(x_{f}-e^{A t_{f}} x_{o}\right)^{\top} W_{r}^{-1}\left(t_{f}\right)\left(x_{f}-e^{A t_{f}} x_{o}\right) \\
& =x_{f}^{\top} W_{r}^{-1}\left(t_{f}\right) x_{f}+x_{o}^{\top} W_{c}^{-1}\left(t_{f}\right) x_{o}-x_{o}^{\top} e^{A t_{f}} W_{r}^{-1}\left(t_{f}\right) x_{f}-x_{f}^{\top} W_{r}^{-1}\left(t_{f}\right) e^{A t_{f}} x_{o} .
\end{aligned}
$$

The expression (3.9) simplifies if either $x_{o}$ or $x_{f}$ is the origin,

1. $x_{o}=0$ (reachability problem)

$$
\Longrightarrow \mathcal{E}_{r}\left(t_{f}\right)=x_{f}^{\top} W_{r}^{-1}\left(t_{f}\right) x_{f}
$$

2. $x_{f}=0$ (controllability to 0 problem)

$$
\Longrightarrow \mathcal{E}_{c}\left(t_{f}\right)=x_{o}^{\top} W_{c}^{-1}\left(t_{f}\right) x_{o} .
$$

For $A$ stable the reachability Gramian converges as $t_{f} \rightarrow \infty$ to the solution of the Lyapunov equation

$$
A^{\top} W_{r}+W_{r} A+B B^{\top}=0
$$

while for $A$ anti-stable (i.e. $-A$ stable) the controllability Gramian converges as $t_{f} \rightarrow \infty$ to the solution of

$$
(-A)^{\top} W_{c}+W_{c}(-A)+B B^{\top}=0 .
$$

Remark 3.1. The controllability literature is not always consistent regarding the definitions of the controllability resp. reachability Gramians. What we call the reachability Gramian is sometimes referred to as the controllability Gramian. The distinction is in many cases irrelevant since for continuous LTI systems the concepts are equivalent. 
Discrete-time LTI systems: Consider the discrete-time LTI system

$$
x(t+1)=A x(t)+B u(t)
$$

where $x(t) \in \mathbb{R}^{n}$ is the state at time $t \in \mathbb{N}_{0}, A \in \mathbb{R}^{n \times n}, B \in \mathbb{R}^{n \times m}$ and $u(t) \in \mathbb{R}^{m}$. The definitions of controllability and reachability are valid also for this system, but there are a few differences as compared to the continuous-time case that need to be pointed out. For the discrete-time system (3.13), reachability implies controllability, but the reverse is not true (this is related to cases where the $A$ matrix is not invertible). Since reachability is the stronger system quality, it is normally of greater interest. The time-invariant linear state equation is reachable on $\left[0, t_{f}\right], t_{f} \in \mathbb{N}$, if and only if the matrix

$$
\mathcal{C}=\left[B A B \ldots A^{t_{f}-1} B\right]
$$

has full row rank. This is equivalent to that the discrete-time reachability Gramian

$$
W_{r}\left(t_{f}\right)=\sum_{t=0}^{t_{f}-1} A^{t} B B^{\top}\left(A^{\top}\right)^{t}
$$

is positive definite. Unlike the continuous case, reachability can fail due to the size of the time interval $t_{f}$. For $t_{f}>n$ it is enough to consider $\mathcal{C}=\left[B A B \ldots A^{n-1} B\right]$.

In analogy with equations (3.7) through (3.10), we define the energy of the discrete-time control input $u(t)$ as

$$
\mathcal{E}(u)=\sum_{t=0}^{t_{f}-1}\|u(t)\|^{2} .
$$

For the reachability problem, the unique control input that steers the network from $x_{o}=0$ to any $x_{f} \in \mathbb{R}^{n}$ in time $t_{f}$ with minimum energy is

$$
\begin{aligned}
& u^{*}(t):=B^{\top}\left(A^{\top}\right)^{t_{f}-t-1} W_{r}^{-1}\left(t_{f}\right) x_{f}, \text { and } \\
& \mathcal{E}\left(u^{*}\right)=x_{f}^{\top} W_{r}^{-1}\left(t_{f}\right) x_{f} .
\end{aligned}
$$

For $A$ stable the reachability Gramian converges as $t_{f} \rightarrow \infty$ to the solution of

$$
A W_{r} A^{\top}-W_{r}+B B^{\top}=0 .
$$

\subsection{Controllability with constrained inputs}

For LTI systems the control inputs are normally assumed unconstrained, but in many different fields in which controllability of complex networks is studied, the control inputs are intrinsically constrained. Also controllability with constrained inputs has a long history, see Chapter 5 of Jacobson (1977) for a survey. Here we present a key result obtained in Brammer (1972). 
Consider the continuous time system (3.1). The admissible controls are all vector functions $u(t)$ taking value in the control restraint set $\Omega$, i.e.

$$
u(t) \in \Omega \subset \mathbb{R}^{m} .
$$

(With unconstrained controls, $\Omega=\mathbb{R}^{m}$.) Brammer's results apply to the weaker controllability notion known as null-controllability.

Definition 3.3 (Null-controllability). The system (3.1) subject to (3.19) is nullcontrollable if there exists an open set $\Gamma \subseteq \mathbb{R}^{n}$ containing the origin for which any $x_{0} \in \Gamma$ can be controlled to the origin in finite time.

We can think of null-controllability as controllability in a sphere which surrounds the origin, hence controllability implies null-controllability but the converse is usually not true. The following conditions are necessary and sufficient for null-controllability:

Theorem 3.1. (Brammer, 1972) Consider the system (3.1) subject to (3.19) satisfying the following conditions:

i) There exists $u \in \Omega$ satisfying $B u=0$ and

ii) the convex hull of $\Omega$ has nonempty interior in $\mathbb{R}^{m}$.

Then the system is null-controllable if and only if

iii) The matrix $\left[\begin{array}{lllll}B & A B & A^{2} B & \ldots & A^{n-1} B\end{array}\right]$ has rank $n$ and

iv) there is no real left eigenvector $v$ of $A$ s.t. $\langle v, B u\rangle \leq 0 \forall u \in \Omega$.

A real eigenvector denotes an eigenvector associated with a real eigenvalue and $\langle v, B u\rangle$ is the Euclidean inner product of the two vectors $v$ and $B u$. An alternative but equivalent formulation of condition iv) in Theorem 3.1 is:

$\left.i v^{\prime}\right)$ For any real left eigenvector $v$ of $A$ there must be a $u \in \Omega$ such that $\left.\langle v, B u\rangle\right\rangle$ 0 .

It should be pointed out that with constrained control inputs, steering the system in certain directions might rely on the rotation of the unforced system. As a consequence, the time it takes to control the state to the origin can not be chosen arbitrarily.

In Paper A we specialize Brammer's conditions for the case $\Omega=\mathbb{R}_{+}^{m}$, i.e. the control inputs are unilateral.

\subsection{Structural controllability}

Here we present the concept of structural controllability which can be used to solve the minimal controllability problem for complex networks. Structural controllability was introduced already in Lin (1974) and the most important theoretical results dates back to the 70s (Glover and Silverman, 1976; Lin, 1974; 
Shields and Pearson, 1976). However, the concept has regained relevance lately in the context of control of complex networks. A linear time-invariant system with parametrized entries

$$
\dot{x}=A_{\Delta} x+B_{\Delta} u,
$$

is said to be structured if the entries of $A_{\Delta}$ and $B_{\Delta}$ are either fixed zeros or independent parameters (i.e. they are not related by algebraic equations).

Definition 3.4 (Structural controllability). The system (3.20) is structurally controllable if there exist values of the independent parameters for which it is controllable (Lin, 1974).

Controllability is a generic property of a structured system, meaning that it holds true for almost all values of the independent parameters. More precisely, almost all means all values outside a proper algebraic variety of the parameter space (Murota, 1987). The existence of one set of values of the independent parameters for which the system is controllable implies that almost all values of the independent parameters render the system controllable.

Remark 3.2. A structured system is strongly structurally controllable if it is controllable for all values of the independent parameters (Mayeda and Yamada, 1979). However, the cases where strong structural controllability can be determined are quite limited, especially when considering complex networks.

In formulating the conditions for structural controllability it is convenient to introduce the graph $\mathcal{G}\left(A_{\Delta}, B_{\Delta}\right)=(\mathcal{V}, \mathcal{E})$ in which both the states and the inputs are represented with nodes and the edges are given by the non-zero parameters of $A_{\Delta}$ and $B_{\Delta}$,

$$
\begin{aligned}
& \mathcal{V}=\mathcal{V}_{A} \cup \mathcal{V}_{B}, \\
& \mathcal{E}=\mathcal{E}_{A} \cup \mathcal{E}_{B} .
\end{aligned}
$$

Here $\mathcal{V}_{A}=\left\{v_{1}, \ldots, v_{n}\right\}$ is the set of state nodes and $\mathcal{V}_{B}=\left\{v_{n+1}, \ldots, v_{n+m}\right\}$ is the set of input nodes. $\mathcal{E}_{A}=\left\{\left(v_{i}, v_{j}\right) \mid\left(A_{\Delta}\right)_{j i} \neq 0\right\}$ is the set of edges between the state nodes and $\mathcal{E}_{B}=\left\{\left(v_{n+i}, v_{j}\right) \mid\left(B_{\Delta}\right)_{j i} \neq 0, v_{n+i} \in \mathcal{V}_{B}, v_{j} \in \mathcal{V}_{A}\right\}$ is the set of edges from the input nodes to the state nodes.

Definition 3.5. A state node is called inaccessible if there is no directed path reaching it from any of the input nodes.

The in-neighborhood of a set $\mathcal{S} \subset \mathcal{V}$ is the set $T(\mathcal{S}) \subseteq \mathcal{V}$ of all nodes from which there exist an edge to a node in $\mathcal{S}$, i.e. $T(\mathcal{S})=\left\{v_{j} \mid\left(v_{j}, v_{i}\right) \in \mathcal{E}, v_{i} \in \mathcal{S}\right\}$. We use $|\mathcal{S}|$ and $|T(\mathcal{S})|$ for the cardinality of $\mathcal{S}$ and $T(\mathcal{S})$ respectively.

Definition 3.6. A dilation in the graph $\mathcal{G}=(\mathcal{V}, \mathcal{E})$ is a subset $\mathcal{S} \subset \mathcal{V}$ without root nodes and such that $|T(\mathcal{S})|<|\mathcal{S}|$. 
Theorem 3.2. (Lin, 1974). The system (3.20) is structurally controllable if and only if

i) The graph $\mathcal{G}\left(A_{\Delta}, B_{\Delta}\right)$ contains no inaccessible state nodes.

ii) The graph $\mathcal{G}\left(A_{\Delta}, B_{\Delta}\right)$ contains no dilation.

The generic rank of the structured matrix $\left[\begin{array}{ll}A_{\Delta} & B_{\Delta}\end{array}\right]$ is defined to be the maximum rank that $\left[\begin{array}{ll}A_{\Delta} & B_{\Delta}\end{array}\right]$ can attain as a function of all the free parameters. The graphical condition ii) of Theorem 3.2 is equivalent to the algebraic condition

ii') The generic rank of $\left[\begin{array}{ll}A_{\Delta} & B_{\Delta}\end{array}\right]=n$.

In the following, conditions i) and ii) of Theorem 3.2 will be referred to as the input connected condition and the rank condition.

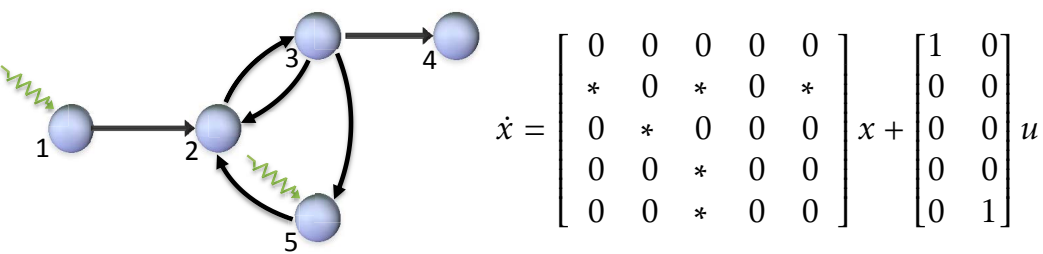

Figure 3.1: The green arrows in the graph indicate control inputs, hence $v_{1}$ and $v_{5}$ are driver nodes. There is a path from the control input applied in $v_{1}$ to all nodes, hence the input connected condition of Theorem 3.2 is met. Furthermore, the control input applied to $v_{5}$ is needed in order to meet the rank condition of Theorem 3.2 because without it, the set of nodes $\mathcal{S}=$ $\left\{v_{4}, v_{5}\right\}$ with in-neighborhood set $T(\mathcal{S})=\left\{v_{3}\right\}$ is a dilation. The generic rank of $\left[A_{\Delta} B_{\Delta}\right]=n$.

\subsection{Minimal controllability of complex networks}

The problem of finding a minimal set of driver nodes that render a given network (structurally) controllable is referred to as the minimal controllability problem (Olshevsky, 2014; Pequito et al., 2014). This problem appears if for instance a cost is associated with the construction of each control input. Moreover, the cardinality of such set, the minimal number of driver nodes, can be regarded a measure of the difficulty to control the network. Given a topological LTI network model where the locations of the edges connecting the nodes are known but not the values of the edge weights, the structural controllability framework offers efficient graph-based methods to solve the minimal controllability problem.

For the system (3.20), represented with the graph $\mathcal{G}\left(A_{\Delta}, B_{\Delta}\right)=(\mathcal{V}, \mathcal{E})$, if the generic rank of $A_{\Delta}$ is $\gamma$, then the generic rank of $B_{\Delta}$ must be at least $n-\gamma$ for the rank condition ii) to hold. The number $\mu_{0}=n-\gamma$, sometimes referred to as the rank deficiency of the network, is the (generic) dimension of the nullspace 
of $A_{\Delta}$ and the minimum number of driver nodes that are necessary to satisfy the rank condition. The generic rank of $A_{\Delta}$ can be computed using for instance the Dulmage-Mendelsohn (DM) decomposition as in Commault and Dion (2013). Besides the rank deficiency of the network, the DM decomposition will also identify driver node candidates such that the rank condition for structural controllability is met.

We call a strongly connected component of $\mathcal{G}\left(A_{\Delta}, B_{\Delta}\right)$ that has no incoming edge from any other strongly connected component a Rooted Connected Component (RCC). The input connected condition for structural controllability is met when all RCCs of the graph $\mathcal{G}\left(A_{\Delta}, B_{\Delta}\right)$ are single input nodes. By combining the DM-decomposition for the rank condition and other graph algorithms for the identification of RCCs, a method for finding a minimal set of driver nodes in polynomial time can be obtained. As will be seen in Paper A, the input connected condition is in many cases met already with the driver nodes that are placed for the rank condition, meaning that the minimal number of driver nodes becomes $\mu_{0}$, the dimension of the null space of $A_{\Delta}$.

Although the minimal controllability problem refers to the placement of driver nodes for controllability, also edge additions can render a network controllable. Moreover, when structural controllability is considered the analysis is quite similar: If the rank condition fails, i.e. the generic rank of $\left[A_{\Delta} B_{\Delta}\right]<n$, then clearly this can be handled by additional driver nodes (columns in $B_{\Delta}$ ), but also by edge additions (elements in $A_{\Delta}$ ). Graph theoretic methods and the DM decomposition can be used also for this case (Chen et al., 2018; Pichai et al., 1981).

\subsection{Minimum energy control of complex networks}

The cost for controlling a network is in many cases related to the energy that the driver nodes must exert. In other cases, the control energy must be limited due to capacity constraints in the actuators or because the network model is otherwise invalid. While equation (3.9) gives the minimal control energy that is needed to achieves a specific state transition, comparing the control energy for different networks and sets of driver nodes in more general ways requires other metrics.

For an LTI network model, if $x_{f}$ is a unit-length eigenvector of the Gramian $W_{r}$, then from (3.10) the minimum energy needed for the state-transition from $x(0)=0$ to $x\left(t_{f}\right)=x_{f}$ is $x_{f}^{\top} W_{r}^{-1} x_{f}=1 / \lambda$, where $\lambda$ is the eigenvalue associated with $x_{f}$ (Yan et al., 2015). For this reason, several metrics for the control energy are based on the eigenvalues $\lambda_{1}, \ldots, \lambda_{n}$ of the Gramian, for instance:

i) $\lambda_{\min }\left(W_{r}\right)=\min \left\{\lambda_{1}, \ldots, \lambda_{n}\right\}$ : The energy required to steer the system from the origin along the worst case direction of the state-space is $1 / \lambda_{\min }\left(W_{r}\right)$.

ii) $\operatorname{Tr}\left(W_{r}^{-1}\right)=\sum_{i=1}^{n} 1 / \lambda_{i}$ : The trace of the inverse Gramian is proportional to the average energy required to control a system in different directions of the state space.

iii) $\operatorname{Tr}\left(W_{r}\right)=\sum_{i=1}^{n} \lambda_{i}$ : The trace of the Gramian is inversely related to the average energy required to control a system, hence when $\operatorname{Tr}\left(W_{r}\right)$ increases the 
control energy decreases. Note that $W_{r}$ may be singular (and the system not controllable) although $\operatorname{Tr}\left(W_{r}\right)$ is high.

iv) $\operatorname{det}\left(W_{r}\right)=\prod_{i=1}^{n} \lambda_{i}$ : The volume of the subset of the state space which is reachable from the origin given a fixed amount of control energy is a function of $\operatorname{det}\left(W_{r}\right)$.

See for instance Müller and Weber (1972); Summers et al. (2016) for a thorough description of these and other metrics for the control energy.

With only a minimal set of driver nodes for controllability it turns out that for instance $\lambda_{\min }\left(W_{r}\right)$ is often very close to zero, meaning that large amounts of control energy, sometimes unreasonable amounts, are required to steer the network in the worst case direction. From (3.4), the Gramian $W_{r}$ is a function of the network adjacency matrix $A$ and the input matrix $B$. Since the metrics i) through iv) are all based on $W_{r}$, they too are functions of $A$ and $B$ and can be improved by the placement of additional driver nodes and edges. The question "How should a limited number of driver nodes/edge additions be placed to obtain the lowest possible energy needed for control?" has no clear answer although several different approaches have been proposed. It is for instance formulated as an optimization problem for driver nodes in Summers et al. (2016); Tzoumas et al. (2016) and for edge manipulations in Becker et al. (2020). Another way to approach the problem is to quantify the importance of the different nodes and edges for controllability with network centrality measures (Bof et al., 2017; Chanekar et al., 2019; Pasqualetti et al., 2014), see also Papers B, C and D. 



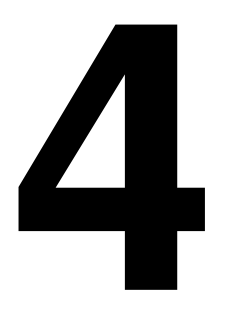

\section{Norms of systems: $\mathcal{H}_{2}$ and $\mathcal{H}_{\infty}$}

In Paper D we use system norms to quantify the impact of edge modifications in a network. While this is interesting in itself, such impact is also related to the controllability of the network. Here we summarize a few properties of the $\mathcal{H}_{2}$ and $\mathcal{H}_{\infty}$ system norms which are used in Part II of the thesis. For a more thorough presentation of the topic, see e.g. Zhou et al. (1996).

A norm is a function defined on a vector space that satisfies conditions on nonnegativity, homogeneity and the triangle inequality. Usually, a norm represents a notion of size. For instance, the $\mathcal{L}_{2}$ signal norm for a transient signal $u(t)=$ $\left[u_{1}(t), \ldots, u_{m}(t)\right]^{\top}$ is defined by

$$
\|u\|_{\mathcal{L}_{2}}=\left(\int_{0}^{\infty} \sum_{k=1}^{m} u_{i}(t)^{2} d t\right)^{1 / 2}
$$

i.e. the square root of the total energy. Observe that for persistent signals, norms that relate to power rather than energy can be used.

Norms of input-output systems often relate the size of the output signals to the size of the input signals. The $\mathcal{H}_{2}$ norm can be interpreted this way for inputs with flat spectrum. For $G(s)$ the transfer function of a stable, continuous-time LTI system with $m$ inputs and $n$ outputs, the $\mathcal{H}_{2}$ norm is defined

$$
\|G\|_{\mathcal{H}_{2}}=\left(\frac{1}{2 \pi} \int_{-\infty}^{\infty} \operatorname{Tr}\left(G(i \omega) G(i \omega)^{*}\right) d \omega\right)^{1 / 2},
$$

where $G(i \omega)^{*}$ denotes the complex conjugate transpose of $G(i \omega)$. Straightforward manipulations of (4.2) gives that $\|G\|_{\mathcal{H}_{2}}^{2}$ is the power of the system outputs when 
white noise with unit variance is applied to the inputs (Zhou et al., 1996). Moreover, it holds

$$
\|G\|_{\mathcal{H}_{2}}=\left(\sum_{k=1}^{n} \sum_{\ell=1}^{m}\left\|G_{k \ell}\right\|_{\mathcal{H}_{2}}^{2}\right)^{1 / 2},
$$

i.e. the root of the sum of the squares of its entities.

With $g(t)$ the impulse response of the system, by Parseval's theorem the $\mathcal{H}_{2}$ norm can be expressed as

$$
\|G\|_{\mathcal{H}_{2}}=\left(\int_{0}^{\infty} \operatorname{Tr}\left(g(t) g(t)^{\top}\right) d t\right)^{1 / 2} .
$$

For the case that $G$ has only one input, the squared $\mathcal{H}_{2}$ norm can be interpreted as the energy (the squared $\mathcal{L}_{2}$ norm) of the impulse response.

Let the matrices $(A, B, C)$ define a state space representation of $G$. Then substituting $g(t)=C e^{A t} B$ into (4.4) gives

$$
\begin{aligned}
\|G\|_{\mathcal{H}_{2}}^{2} & =\int_{0}^{\infty} \operatorname{Tr}\left(C e^{A t} B\left(C e^{A t} B\right)^{\top}\right) d t=\operatorname{Tr}\left(C\left(\int_{0}^{\infty} e^{A t} B B^{\top} e^{A^{\top} t} d t\right) C^{\top}\right) \\
& =\operatorname{Tr}\left(C W_{r} C^{\top}\right),
\end{aligned}
$$

with $W_{r}$ the infinite time reachability Gramian. In fact, the $\mathcal{H}_{2}$ norm is usually computed by first solving the Lyapunov equation (3.11) for $W_{r}$ and then using (4.5). Furthermore, from (4.5) we see that the control energy metric $\operatorname{Tr}\left(W_{r}\right)$ for the network model (1.1) coincides with the squared $\mathcal{H}_{2}$ norm when $C$ is the identity matrix, i.e. the state of each node is considered an output.

The $\mathcal{H}_{\infty}$ norm is induced by the $\mathcal{L}_{2}$ signal norm, meaning that it is the largest ratio of of the $\mathcal{L}_{2}$ norm of the output signal to the $\mathcal{L}_{2}$ norm of the input signal,

$$
\|G\|_{\mathcal{H}_{\infty}}=\sup _{u(t)} \frac{\|G u(t)\|_{\mathcal{L}_{2}}}{\|u(t)\|_{\mathcal{L}_{2}}} .
$$

Clearly, $\|G\|_{\mathcal{H}_{\infty}}<\infty$ if and only if $G$ is stable, in which case the norm can be expressed as the maximum of the largest singular value of the frequency function over all frequencies:

$$
\|G\|_{\mathcal{H}_{\infty}}=\sup _{\omega} \sigma_{\max }(G(i \omega))
$$

The $\mathcal{H}_{\infty}$ norm cannot usually be computed directly for LTI systems, instead, one has to test if $\|G\|_{\mathcal{H}_{\infty}}<\gamma$ for some $\gamma>0$. 


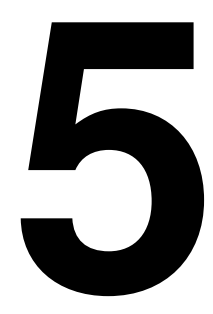

\section{Concluding remarks}

In an effort to render more realistic the problem of controlling complex networks, in the first paper we study the minimal controllability problem given a network model with linear dynamics but unilateral control inputs. The model is motivated by the fact that unilateral controls are more common than bidirectional controls in many contexts. To enable a large scale analysis applicable to complex networks, we cast classical controllability results valid in this particular case into a more computationally-efficient form. The obtained controllability conditions are formulated algebraically in terms of the real eigenspaces of the weighted adjacency matrix $A$ and positively spanning sets. With random edge weight assignments, the non-zero eigenvalues of $A$ are generically simple and the null space is determined by the topology of the network. As a result, the unilateral controllability problem is to a high degree a topological problem. Moreover, we have studied various categories of random networks as well as real-world networks using an algorithm that identifies a near minimal set of unilateral control inputs, and found that two factors essentially determines the minimal unilateral controllability problem: the dimension of the null space of $A$ and to what extent the null space is generated by topological roots or dilations. In contrast, the values of the edge weights have only a minor significance. In comparison, the minimal number of unconstrained control inputs is determined by the dimension of the null space of $A$ but does not depend on whether it is roots or dilations that generate it. The difference is considerably large for some classes of networks, almost twice as many unilateral control inputs as unconstrained are needed for networks dominated by hubs with high indegree. However, for most networks the difference is much smaller and for networks dominated by hubs with high outdegree it is negligible.

The relevance of "theoretical controllability" with its "yes" or "no" answer, has however several shortcomings. On the one hand it can be argued that con- 
trollability is an unnecessarily ambitious goal. In many applications it is enough to control a subset of the nodes, i.e. achieve controllability in a subspace of $\mathbb{R}^{n}$. On the other hand, a network that is controllable according to the mathematical definition is often not controllable in practice due to unrealistic requirements on the control energy. This is studied in the Papers B and C, now under the assumption that the control inputs are unconstrained. In Paper B we adopt an LTI network model and allow networks with both stable and unstable eigenmodes. By analyzing numerically how the location of the eigenvalues of the weighted adjacency matrix $A$ influence the reachability Gramian and the energy measures that can be obtained from it, we find that the time constants of the eigenmodes of $A$ play a key role regardless of the number of driver nodes (driver nodes are randomly placed and controllability is ensured). For controllability to the origin, the control inputs must "dominate" the eigenmodes of eigenvalues with positive real part, while for controllability from the origin the eigenmodes of eigenvalues with negative real part are the more difficult ones to handle. Generally, the energy required for control increases with the magnitudes of the real parts of the eigenvalues. For the special case with purely imaginary spectra we propose three different algorithms for the placement of driver nodes, each one designed for the optimization of one specific energy measure. For the general case with networks with arbitrary eigenvalues, Paper B suggests a strategy for the placement of driver nodes that consists in ranking the nodes by a connectivity property expressed as the ratio between the weighted outdegree and the weighted indegree of the nodes. The strategy outperforms a random placement of equally many driver nodes, and the improvements increases with the connectivity ratio. For instance, the worstcase control energy for directed scale free networks is reduced by several orders of magnitude.

The driver node placement strategy suggested in Paper B based on a numerical analysis is further developed and theoretically investigated in Paper C. A key observation for placement of driver nodes is that what makes a good driver node depends both on its influence over other nodes in the network and on its ability to be controlled indirectly from other nodes. In Paper $\mathrm{C}$ we utilize this and propose the use of two network centrality measures, the node-to-network centrality $p$ and the network-to-node centrality $q$, for the placement of driver nodes. They are both based on energy flow considerations: $p_{i}$ is the energy flow from node $v_{i}$ to all nodes in the network and $q_{i}$ is the energy flow from all nodes to $v_{i}$. The node-to-network centrality reflects the network impact of a node, in fact, a driver node placement based on $p$ optimizes the trace of the reachability Gramian control energy metric. The network-to-node centrality instead quantifies the ability to control a node indirectly from the other nodes. It is in a sense an extension of the rank condition for structural controllability, which requires nodes that cannot be indirectly controlled to be driver nodes themselves. Low $q_{i}$ corresponds to nodes that "almost" cannot be controlled indirectly. Moreover, the distribution of $q$ for the nodes in a network is formally related to both the worst-case control energy and the average control energy.

Based on the notion of balanced realization, our characterization of network non-normality as imbalances in the distribution of energy over the network al- 
lows us to quantify non-normality at a node-level in terms of the network-tonode and node-to-network centralities. Selecting the nodes with maximal nonnormality as driver nodes results in reduced energy requirements for controlling the network, i.e. both the average energy needed to steer the state in any direction and the energy needed for the worst direction are simultaneously improved w.r.t. a random driver node placement. As with the ranking suggested in Paper $\mathrm{B}$, to which the current rankings are closely related, the improvements are visible but limited for Erdős-Rényi networks, but significantly higher, approx. 3 orders of magnitude, for the more non-normal directed scale-free networks.

Compared to the placement of driver nodes, relatively little has been written about how edge additions can be designed to render a network controllable. In Paper D we address this problem by first deriving a sensitivity function for edge modifications in a network and then quantifying the effects of edge additions with the $\mathcal{H}_{2}$ and $\mathcal{H}_{\infty}$ norms. For networks with positive edge weights we show how the $\mathcal{H}_{\infty}$ norm can be computed exactly for each possible edge modification, while for the $\mathcal{H}_{2}$ norm we instead obtain a lower bound. In simulations, however, this bound proves to be a very good approximation of the exact $\mathcal{H}_{2}$ norm. The bound can also be used to optimize certain control energy metrics, in fact, its expression involves variants of both the node-to-network and the network-to-node centralities, pointing to both similarities and differences between driver node placement and edge additions for controllability.

There is still much to investigate regarding the design of edge additions for controllability and we believe that there is a large potential in it. For instance, our results in Paper D suggests that methods originally developed for driver node placement in complex networks may be possible to adapt to consider also edge modifications. 



\section{Bibliography}

P.J. Antsaklis and A.N. Michel. Linear Systems. Birkhäuser Boston, 2005.

Albert-László Barabási and Réka Albert. Emergence of scaling in random networks. Science, 286(5439):509-512, 1999.

Cassiano O Becker, Sérgio Pequito, George J Pappas, and Victor M Preciado. Network design for controllability metrics. 2020.

Nicoletta Bof, Giacomo Baggio, and Sandro Zampieri. On the role of network centrality in the controllability of complex networks. IEEE Transactions on Control of Network Systems, 4(3):643-653, 2017.

Robert F. Brammer. Controllability in linear autonomous systems with positive controllers. SIAM Journal on Control, 10(2):339-353, 1972.

Francesca Ceragioli, Gustav Lindmark, Clas Veibäck, Niklas Wahlström, Martin Lindfors, and Claudio Altafini. A bounded confidence model that preserves the signs of the opinions. In Proceedings of the 2016 European Control Conference (ECC), pages 543-548. IEEE, 2016.

Chanekar, Nozari, and Cortés. Network modification using a novel gramianbased edge centrality. In 2019 58th IEEE Conference on Decision and Control (CDC). IEEE, 2019.

Ximing Chen, Sérgio Pequito, George J Pappas, and Victor M Preciado. Minimal edge addition for network controllability. IEEE Transactions on Control of Network Systems, 6(1):312-323, 2018.

Yu-Zhong Chen, Le-Zhi Wang, Wen-Xu Wang, and Ying-Cheng Lai. Energy scaling and reduction in controlling complex networks. Royal Society Open Science, 3(4), 2016. doi: 10.1098/rsos.160064.

Christian Commault and Jean-Michel Dion. Input addition and leader selection for the controllability of graph-based systems. Automatica, 49(11):3322-3328, 2013. ISSN 0005-1098.

K. Glover and L. Silverman. Characterization of structural controllability. IEEE Transactions on Automatic control, 21(4):534-537, 1976. 
D.H. Jacobson. Extensions of Linear-Quadratic Control, Optimization and Matrix Theory, volume 133 of Mathematics in Science and Engineering. Academic Press, London, 1977.

Rudolf Emil Kalman. Mathematical description of linear dynamical systems. Journal of the Society for Industrial \& Applied Mathematics, Series A: Control, 1(2):152-192, 1963.

Guoqi Li, Wuhua Hu, Gaoxi Xiao, Lei Deng, Pei Tang, Jing Pei, and Luping Shi. Minimum-cost control of complex networks. New Journal of Physics, 18(1): $013012,2016$.

Ching Tai Lin. Structural controllability. IEEE Transactions on Automatic Control, 19(3):201-208, 1974.

Gustav Lindmark and Claudio Altafini. Positive controllability of large-scale networks. In Proceedings of the 2016 European Control Conference (ECC), pages 819-824. IEEE, 2016.

Gustav Lindmark and Claudio Altafini. Controllability of complex networks with unilateral inputs. Scientific Reports, 7:1824, 2017a. doi: 10.1038/ s41598-017-01846-6.

Gustav Lindmark and Claudio Altafini. A driver node selection strategy for minimizing the control energy in complex networks. In Proceedings of the 2017 IFAC World Congress, volume 50, pages 8309-8314. Elsevier, 2017b.

Gustav Lindmark and Claudio Altafini. Minimum energy control for networks of coupled harmonic oscillators. In Proceedings of the 2017 IFAC World Congress, volume 50, pages 8321-8326. Elsevier, 2017c.

Gustav Lindmark and Claudio Altafini. Topological aspects of controlling large scale networks with unilateral inputs. In Proceedings of the 2017 IFAC World Congress, volume 50, pages 8315-8320. Elsevier, 2017d.

Gustav Lindmark and Claudio Altafini. Minimum energy control for complex networks. Scientific Reports, 8(1):3188, 2018.

Gustav Lindmark and Claudio Altafini. Combining centrality measures for control energy reduction in network controllability problems. In 2019 European Control Conference (ECC), pages 1518-1523. IEEE, 2019.

Gustav Lindmark and Claudio Altafini. On the impact of edge modifications for networked control systems. In Proceedings of the 2020 IFAC World Congress, to appear, 2020a.

Gustav Lindmark and Claudio Altafini. Centrality measures and the role of nonnormality for network control energy reduction. Preprint, 2020 b.

Yang-Yu Liu, Jean-Jacques Slotine, and Albert-László Barabási. Control centrality and hierarchical structure in complex networks. Plos one, 7(9):e44459, 2012. 
Charles P. Madenjian et al. Dynamics of the lake michigan food web, 1970-2000. Canadian Journal of Fisheries and Aquatic Sciences, 59(4):736-753, 2002.

Iven Mareels, Erik Weyer, Su Ki Ooi, Michael Cantoni, Yuping Li, and Girish Nair. Systems engineering for irrigation systems: Successes and challenges. Annual reviews in control, 29(2):191-204, 2005.

Hirokazu Mayeda and Takashi Yamada. Strong structural controllability. SIAM Journal on Control and Optimization, 17(1):123-138, 1979.

P.C. Müller and H.I. Weber. Analysis and optimization of certain qualities of controllability and observability for linear dynamical systems. Automatica, 8(3):237 - 246, 1972. ISSN 0005-1098. doi: http://dx.doi.org/10.1016/ 0005-1098(72)90044-1.

Kazuo Murota. Systems analysis by graphs and matroids. In Algorithms and Combinatorics, volume 3. Springer Verlag Berlin, 1987.

Jose C. Nacher and Tatsuya Akutsu. Analysis of critical and redundant nodes in controlling directed and undirected complex networks using dominating sets. Journal of Complex Networks, 2(4):394-412, 2014. doi: 10.1093/comnet/ cnu029.

Mark Newman. Networks: an introduction. Oxford University Press, 2010.

A. Olshevsky. Minimal controllability problems. IEEE Transactions on Control of Network Systems, 1(3):249-258, Sept 2014. ISSN 2325-5870. doi: 10.1109/ TCNS.2014.2337974.

Alex Olshevsky. Eigenvalue clustering, control energy, and logarithmic capacity. Systems \& Control Letters, 96:45-50, 2016.

Fabio Pasqualetti, Sandro Zampieri, and Francesco Bullo. Controllability metrics, limitations and algorithms for complex networks. IEEE Transactions on Control of Network Systems, 1(1):40-52, 2014.

Sergio Pequito, Guilherme Ramos, Soummya Kar, A. Pedro Aguiar, and Jaime Ramos. On the exact solution of the minimal controllability problem. arXiv preprint:1401.4209, 2014.

V Pichai, ME Sezer, and DD Šiljak. Vulnerability of dynamic systems. International Journal of Control, 34(6):1049-1060, 1981.

Wilson J. Rugh. Linear system theory. Prentice Hall, Inc., second edition, 1996.

Robert Shields and J. Pearson. Structural controllability of multi-input linear systems. IEEE Transactions on Automatic control, 21(2):203-212, 1976.

Eduardo D. Sontag. Mathematical control theory: deterministic finite dimensional systems, volume 6. Springer Science \& Business Media, 2013. 
Tyler H. Summers, Fabrizio L. Cortesi, and John Lygeros. On submodularity and controllability in complex dynamical networks. IEEE Transactions on Control of Network Systems, 3(1):91-101, 2016.

Girma S. Tewolde. Sensor and network technology for intelligent transportation systems. In Electro/Information Technology (EIT), 2012 IEEE International Conference on, pages 1-7. IEEE, 2012.

Núria Ballber Torres and Claudio Altafini. Drug combinatorics and side effect estimation on the signed human drug-target network. BMC Systems Biology, 10(1):74, Aug 2016. ISSN 1752-0509. doi: 10.1186/s12918-016-0326-8. URL https://doi.org/10.1186/s12918-016-0326-8.

Vasileios Tzoumas, Mohammad Amin Rahimian, George J. Pappas, and Ali Jadbabaie. Minimal actuator placement with bounds on control effort. IEEE Transactions on Control of Network Systems, 3(1):67-78, 2016.

Gang Yan, Jie Ren, Ying-Cheng Lai, Choy-Heng Lai, and Baowen Li. Controlling complex networks: How much energy is needed? Physical Review Letters, 108: 218703, May 2012. doi: 10.1103/PhysRevLett.108.218703.

Gang Yan, Georgios Tsekenis, Baruch Barzel, Jean-Jacques Slotine, Yang-Yu Liu, and Albert-László Barabási. Spectrum of controlling and observing complex networks. Nature Physics, 11(9):779-786, 2015.

Kemin Zhou, John Comstock Doyle, Keith Glover, et al. Robust and optimal control, volume 40. Prentice Hall New Jersey, 1996. 


\section{Part II}

\section{Publications}





\section{Papers}

The papers associated with this thesis have been removed for copyright reasons. For more details about these see:

http://urn.kb.se/resolve?urn=urn:nbn:se:liu:diva-165258 


\section{PhD Dissertations \\ Division of Automatic Control \\ Linköping University}

M. Millnert: Identification and control of systems subject to abrupt changes. Thesis No. 82, 1982. ISBN 91-7372-542-0.

A. J. M. van Overbeek: On-line structure selection for the identification of multivariable systems. Thesis No. 86, 1982. ISBN 91-7372-586-2.

B. Bengtsson: On some control problems for queues. Thesis No. 87, 1982. ISBN 91-7372593-5.

S. Ljung: Fast algorithms for integral equations and least squares identification problems. Thesis No. 93, 1983. ISBN 91-7372-641-9.

H. Jonson: A Newton method for solving non-linear optimal control problems with general constraints. Thesis No. 104, 1983. ISBN 91-7372-718-0.

E. Trulsson: Adaptive control based on explicit criterion minimization. Thesis No. 106, 1983. ISBN 91-7372-728-8.

K. Nordström: Uncertainty, robustness and sensitivity reduction in the design of single input control systems. Thesis No. 162, 1987. ISBN 91-7870-170-8.

B. Wahlberg: On the identification and approximation of linear systems. Thesis No. 163, 1987. ISBN 91-7870-175-9.

S. Gunnarsson: Frequency domain aspects of modeling and control in adaptive systems. Thesis No. 194, 1988. ISBN 91-7870-380-8.

A. Isaksson: On system identification in one and two dimensions with signal processing applications. Thesis No. 196, 1988. ISBN 91-7870-383-2.

M. Viberg: Subspace fitting concepts in sensor array processing. Thesis No. 217, 1989. ISBN 91-7870-529-0.

K. Forsman: Constructive commutative algebra in nonlinear control theory. Thesis No. 261, 1991. ISBN 91-7870-827-3.

F. Gustafsson: Estimation of discrete parameters in linear systems. Thesis No. 271, 1992. ISBN 91-7870-876-1.

P. Nagy: Tools for knowledge-based signal processing with applications to system identification. Thesis No. 280, 1992. ISBN 91-7870-962-8.

T. Svensson: Mathematical tools and software for analysis and design of nonlinear control systems. Thesis No. 285, 1992. ISBN 91-7870-989-X.

S. Andersson: On dimension reduction in sensor array signal processing. Thesis No. 290, 1992. ISBN 91-7871-015-4.

H. Hjalmarsson: Aspects on incomplete modeling in system identification. Thesis No. 298, 1993. ISBN 91-7871-070-7.

I. Klein: Automatic synthesis of sequential control schemes. Thesis No. 305, 1993. ISBN 91-7871-090-1.

J.-E. Strömberg: A mode switching modelling philosophy. Thesis No. 353, 1994. ISBN 917871-430-3.

K. Wang Chen: Transformation and symbolic calculations in filtering and control. Thesis No. 361, 1994. ISBN 91-7871-467-2.

T. McKelvey: Identification of state-space models from time and frequency data. Thesis No. 380, 1995. ISBN 91-7871-531-8.

J. Sjöberg: Non-linear system identification with neural networks. Thesis No. 381, 1995. ISBN 91-7871-534-2.

R. Germundsson: Symbolic systems - theory, computation and applications. Thesis No. 389, 1995. ISBN 91-7871-578-4. 
P. Pucar: Modeling and segmentation using multiple models. Thesis No. 405, 1995. ISBN 91-7871-627-6.

H. Fortell: Algebraic approaches to normal forms and zero dynamics. Thesis No. 407, 1995. ISBN 91-7871-629-2.

A. Helmersson: Methods for robust gain scheduling. Thesis No. 406, 1995. ISBN 91-7871628-4.

P. Lindskog: Methods, algorithms and tools for system identification based on prior knowledge. Thesis No. 436, 1996. ISBN 91-7871-424-8.

J. Gunnarsson: Symbolic methods and tools for discrete event dynamic systems. Thesis No. 477, 1997. ISBN 91-7871-917-8.

M. Jirstrand: Constructive methods for inequality constraints in control. Thesis No. 527, 1998. ISBN 91-7219-187-2.

U. Forssell: Closed-loop identification: Methods, theory, and applications. Thesis No. 566, 1999. ISBN 91-7219-432-4.

A. Stenman: Model on demand: Algorithms, analysis and applications. Thesis No. 571, 1999. ISBN 91-7219-450-2.

N. Bergman: Recursive Bayesian estimation: Navigation and tracking applications. Thesis No. 579, 1999. ISBN 91-7219-473-1.

K. Edström: Switched bond graphs: Simulation and analysis. Thesis No. 586, 1999. ISBN 91-7219-493-6.

M. Larsson: Behavioral and structural model based approaches to discrete diagnosis. Thesis No. 608, 1999. ISBN 91-7219-615-5.

F. Gunnarsson: Power control in cellular radio systems: Analysis, design and estimation. Thesis No. 623, 2000. ISBN 91-7219-689-0.

V. Einarsson: Model checking methods for mode switching systems. Thesis No. 652, 2000. ISBN 91-7219-836-2.

M. Norrlöf: Iterative learning control: Analysis, design, and experiments. Thesis No. 653, 2000. ISBN 91-7219-837-0.

F. Tjärnström: Variance expressions and model reduction in system identification. Thesis No. 730, 2002. ISBN 91-7373-253-2.

J. Löfberg: Minimax approaches to robust model predictive control. Thesis No. 812, 2003. ISBN 91-7373-622-8.

J. Roll: Local and piecewise affine approaches to system identification. Thesis No. 802, 2003. ISBN 91-7373-608-2.

J. Elbornsson: Analysis, estimation and compensation of mismatch effects in A/D converters. Thesis No. 811, 2003. ISBN 91-7373-621-X.

O. Härkegård: Backstepping and control allocation with applications to flight control. Thesis No. 820, 2003. ISBN 91-7373-647-3.

R. Wallin: Optimization algorithms for system analysis and identification. Thesis No. 919, 2004. ISBN 91-85297-19-4.

D. Lindgren: Projection methods for classification and identification. Thesis No. 915, 2005. ISBN 91-85297-06-2.

R. Karlsson: Particle Filtering for Positioning and Tracking Applications. Thesis No. 924, 2005. ISBN 91-85297-34-8.

J. Jansson: Collision Avoidance Theory with Applications to Automotive Collision Mitigation. Thesis No. 950, 2005. ISBN 91-85299-45-6.

E. Geijer Lundin: Uplink Load in CDMA Cellular Radio Systems. Thesis No. 977, 2005. ISBN 91-85457-49-3.

M. Enqvist: Linear Models of Nonlinear Systems. Thesis No. 985, 2005. ISBN 91-8545764-7.

T. B. Schön: Estimation of Nonlinear Dynamic Systems - Theory and Applications. Thesis No. 998, 2006. ISBN 91-85497-03-7. 
I. Lind: Regressor and Structure Selection - Uses of ANOVA in System Identification. Thesis No. 1012, 2006. ISBN 91-85523-98-4.

J. Gillberg: Frequency Domain Identification of Continuous-Time Systems Reconstruction and Robustness. Thesis No. 1031, 2006. ISBN 91-85523-34-8.

M. Gerdin: Identification and Estimation for Models Described by Differential-Algebraic Equations. Thesis No. 1046, 2006. ISBN 91-85643-87-4.

C. Grönwall: Ground Object Recognition using Laser Radar Data - Geometric Fitting, Performance Analysis, and Applications. Thesis No. 1055, 2006. ISBN 91-85643-53-X.

A. Eidehall: Tracking and threat assessment for automotive collision avoidance. Thesis No. 1066, 2007. ISBN 91-85643-10-6.

F. Eng: Non-Uniform Sampling in Statistical Signal Processing. Thesis No. 1082, 2007. ISBN 978-91-85715-49-7.

E. Wernholt: Multivariable Frequency-Domain Identification of Industrial Robots. Thesis No. 1138, 2007. ISBN 978-91-85895-72-4.

D. Axehill: Integer Quadratic Programming for Control and Communication. Thesis No. 1158, 2008. ISBN 978-91-85523-03-0.

G. Hendeby: Performance and Implementation Aspects of Nonlinear Filtering. Thesis No. 1161, 2008. ISBN 978-91-7393-979-9.

J. Sjöberg: Optimal Control and Model Reduction of Nonlinear DAE Models. Thesis No. 1166, 2008. ISBN 978-91-7393-964-5.

D. Törnqvist: Estimation and Detection with Applications to Navigation. Thesis No. 1216, 2008. ISBN 978-91-7393-785-6.

P-J. Nordlund: Efficient Estimation and Detection Methods for Airborne Applications. Thesis No. 1231, 2008. ISBN 978-91-7393-720-7.

H. Tidefelt: Differential-algebraic equations and matrix-valued singular perturbation. Thesis No. 1292, 2009. ISBN 978-91-7393-479-4.

H. Ohlsson: Regularization for Sparseness and Smoothness - Applications in System Identification and Signal Processing. Thesis No. 1351, 2010. ISBN 978-91-7393-287-5.

S. Moberg: Modeling and Control of Flexible Manipulators. Thesis No. 1349, 2010. ISBN 978-91-7393-289-9.

J. Wallén: Estimation-based iterative learning control. Thesis No. 1358, 2011. ISBN 978 91-7393-255-4.

J. D. Hol: Sensor Fusion and Calibration of Inertial Sensors, Vision, Ultra-Wideband and GPS. Thesis No. 1368, 2011. ISBN 978-91-7393-197-7.

D. Ankelhed: On the Design of Low Order H-infinity Controllers. Thesis No. 1371, 2011. ISBN 978-91-7393-157-1.

C. Lundquist: Sensor Fusion for Automotive Applications. Thesis No. 1409, 2011. ISBN 978-91-7393-023-9.

P. Skoglar: Tracking and Planning for Surveillance Applications. Thesis No. 1432, 2012. ISBN 978-91-7519-941-2.

K. Granström: Extended target tracking using PHD filters. Thesis No. 1476, 2012. ISBN 978-91-7519-796-8.

C. Lyzell: Structural Reformulations in System Identification. Thesis No. 1475, 2012. ISBN 978-91-7519-800-2.

J. Callmer: Autonomous Localization in Unknown Environments. Thesis No. 1520, 2013. ISBN 978-91-7519-620-6.

D. Petersson: A Nonlinear Optimization Approach to H2-Optimal Modeling and Control. Thesis No. 1528, 2013. ISBN 978-91-7519-567-4.

Z. Sjanic: Navigation and Mapping for Aerial Vehicles Based on Inertial and Imaging Sensors. Thesis No. 1533, 2013. ISBN 978-91-7519-553-7. 
F. Lindsten: Particle Filters and Markov Chains for Learning of Dynamical Systems. Thesis No. 1530, 2013. ISBN 978-91-7519-559-9.

P. Axelsson: Sensor Fusion and Control Applied to Industrial Manipulators. Thesis No. 1585, 2014. ISBN 978-91-7519-368-7.

A. Carvalho Bittencourt: Modeling and Diagnosis of Friction and Wear in Industrial Robots. Thesis No. 1617, 2014. ISBN 978-91-7519-251-2.

M. Skoglund: Inertial Navigation and Mapping for Autonomous Vehicles. Thesis No. 1623, 2014. ISBN 978-91-7519-233-8.

S. Khoshfetrat Pakazad: Divide and Conquer: Distributed Optimization and Robustness Analysis. Thesis No. 1676, 2015. ISBN 978-91-7519-050-1.

T. Ardeshiri: Analytical Approximations for Bayesian Inference. Thesis No. 1710, 2015. ISBN 978-91-7685-930-8.

N. Wahlström: Modeling of Magnetic Fields and Extended Objects for Localization Applications. Thesis No. 1723, 2015. ISBN 978-91-7685-903-2.

J. Dahlin: Accelerating Monte Carlo methods for Bayesian inference in dynamical models. Thesis No. 1754, 2016. ISBN 978-91-7685-797-7.

M. Kok: Probabilistic modeling for sensor fusion with inertial measurements. Thesis No. 1814, 2016. ISBN 978-91-7685-621-5.

J. Linder: Indirect System Identification for Unknown Input Problems: With Applications to Ships. Thesis No. 1829, 2017. ISBN 978-91-7685-588-1.

M. Roth: Advanced Kalman Filtering Approaches to Bayesian State Estimation. Thesis No. 1832, 2017. ISBN 978-91-7685-578-2.

I. Nielsen: Structure-Exploiting Numerical Algorithms for Optimal Control. Thesis No. 1848, 2017. ISBN 978-91-7685-528-7.

D. Simon: Fighter Aircraft Maneuver Limiting Using MPC: Theory and Application. Thesis No. 1881, 2017. ISBN 978-91-7685-450-1.

C. Veibäck: Tracking the Wanders of Nature. Thesis No. 1958, 2018. ISBN 978-91-7685200-2.

C. Andersson Naesseth: Machine learning using approximate inference: Variational and sequential Monte Carlo methods. Thesis No. 1969, 2018. ISBN 978-91-7685-161-6.

Y. Jung: Inverse system identification with applications in predistortion. Thesis No. 1966, 2018. ISBN 978-91-7685-171-5.

Y. Zhao: Gaussian Processes for Positioning Using Radio Signal Strength Measurements. Thesis No. 1968, 2019. ISBN 978-91-7685-162-3.

R. Larsson: Flight Test System Identification. Thesis No. 1990, 2019. ISBN 978-91-7685070-1.

P. Kasebzadeh: Learning Human Gait. Thesis No. 2012, 2019. ISBN 978-91-7519-014-3.

K. Radnosrati: Time of Flight Estimation for Radio Network Positioning. Thesis No. 2054, 2020. ISBN 978-91-7929-884-5.

O. Ljungqvist: Motion planning and feedback control techniques with applications to long tractor-trailer vehicles. Thesis No. 2070, 2020. ISBN 978-91-7929-858-6. 


\section{FACULTY OF SCIENCE AND ENGINEERING}

Linköping Studies in Science and Technology, Dissertation No. 2074, 2020

Department of Electrical Engineering

Linköping University

SE-581 83 Linköping, Sweden

www.liu.se 